Aproximación a la producción alfarera en los sitios Alamito durante los primeros siglos d.C. (Noroeste argentino)

Lucas Pereyra Domingorena

Relaciones, 46(2), e 018, julio-diciembre 2021

ISSN 1852-1479 | https://doi.org/10.24215/18521479e018

https://revistas.unlp.edu.ar/relaciones

ISSN 0325-2221 (versión impresa)

Sociedad Argentina de Antropología (SAA)

Buenos Aires I Argentina

\title{
APROXIMACIÓN A LA PRODUCCIÓN ALFARERA EN LOS SITIOS ALAMITO DURANTE LOS PRIMEROS SIGLOS D.C. (NOROESTE ARGENTINO)
}

Lucas Pereyra Domingorena*

Fecha de recepción: 14 de enero de 2021

Fecha de aceptación: 7 de octubre de 2021

\section{RESUMEN}

Esta investigación procura entender la producción alfarera en los sitios Alamito (Noroeste argentino), habitados durante los primeros siglos $d . C$. Conocer esta manufactura artesanal es adentrarse en las dinámicas sociales de estos grupos humanos que se caracterizaron por una particular forma de construir, distribuir en el paisaje y abandonar sus viviendas. Se seleccionaron 55 tiestos -representan la variabilidad estilística-morfológica de la muestra estudiada- y fueron sometidos al análisis petrográfico de sus pastas cerámicas. La metodología utilizada incluye el estudio de la matriz arcillosa, la clasificación de las inclusiones (tipo, tamaño y formas) y la descripción de las cavidades. Las regularidades y particularidades registradas en los datos han permitido detectar seis grupos de pastas, que fueron comparados con modos de pastas de áreas cercanas. Así, este trabajo analizó las relaciones entre la cerámica Alamito y otras producciones cerámicas del Noroeste argentino durante el Período Formativo.

Palabras clave: Noroeste argentino - Período Formativo-Alamito-alfarería-petrografía.

\footnotetext{
* Instituto de las Culturas (IDECU)-Universidad de Buenos Aires-CONICET, Facultad de Filosofía y Letras, Museo Etnográfico “Juan B. Ambrosetti”. E-mail: lucasdomingorena@gmail.com
} 
Relaciones de la Sociedad Argentina de Antropología 46 (2), julio-diciembre 2021: 503-525

\author{
POTTERY PRODUCTION AT ALAMITO SITES \\ DURING THE FIRST CENTURIES AD (NORTHWEST ARGENTINA)
}

\title{
ABSTRACT
}

The goal of this research is to understand pottery production in the Alamito sites (northwestern Argentina), inhabited during the first centuries AD. To know this craft production is to enter into the social dynamics of these human groups characterized by a particular way of building, distributing in the landscape and abandoning their dwellings. 55 sherds representative of the stylistic-morphological variability of the sample under study were selected and their fabrics were assessed using petrographic analysis. The methodology used includes the study of the clay matrix, classification of inclusions (type, size and shapes) and description of cavities. The regularities and singularities observed in this data allowed to detect six classes of fabric, which were compared with fabric recipes from nearby areas. Thus, this paper analyzed the relationships between the Alamito pottery and other ceramic productions of northwestern Argentina during the Formative Period.

Keywords: Argentine Northwest - Formative Period - Alamito - pottery - petrography

\section{INTRODUCCIÓN}

Este trabajo procura comprender la producción alfarera de los grupos humanos que habitaron los sitios Alamito durante los primeros siglos d.C., en la región de Campo del Pucará (provincia de Catamarca, Argentina) (figura 1). Las manufacturas, en este caso la alfarería, son parte constitutiva de las sociedades humanas, conforman un savoir faire o maniera de hacer objetos que implica las técnicas y sus procesos de producción, es decir, está compuesto tanto por las materias primas, las fuentes de energía, las herramientas y la planificación (Stark 1999) -necesarias para la confección de un determinado bien-, así como también por las habilidades incorporadas y el conocimiento práctico (Mauss 1979 [1935], Lemonnier 2002 [1993]) que deben tener las artesanas y artesanos. Además, se piensa que en las sociedades de pequeña escala-como las aquí analizadas- esta cognición práctica se proyecta también como un medio para la transmisión del conocimiento social. En particular, las evidencias etnográficas sobre la transmisión de rasgos culturales en la producción cerámica han detectado que ésta conlleva creencias espirituales, ordenamiento mítico, ideas sobre diferencias de identidad, etc. (Miller 1985; Gosselain 1992, 1999; Hosler 1996). Las dimensiones económicas, sociales, rituales, mágicas y políticas de los procesos técnicos están mayormente expresadas en metáforas que dan sentido al mundo (Lemonnier 2002 [1993]; Tilley 1999; Stark 1999). Los objetos así elaborados sirven como fuente de representaciones para la constitución de la identidad social, por la utilización diferencial de tales cosas según dimensiones como el sexo, la edad o la posición en el entramado social (Bourdieu 2007 [1980]; Tilley 1999). Además, la importancia cultural de los estilos técnicos se manifestaría fuertemente en sociedades orales, como las que habitaron Campo del Pucará durante los primeros siglos d.C. El traspaso generacional de estos saberes sobre el uso de materias primas y herramientas, las secuencias operativas y las habilidades ofrecen al pensamiento oral un anclaje material útil para su transmisión a lo largo del tiempo, ya que está fuertemente relacionado con el mundo vital humano (Ong 2006 [1982]).

Los estilos técnicos serían, entonces, parte del andamiaje dóxico de las sociedades, es decir, de ese "conjunto de presupuestos inseparablemente cognitivos y evaluativos cuya aceptación resulta implícita por el hecho de pertenecer a él” (Bourdieu 1999 [1997]:134). Los sujetos sociales están inmersos en esos conceptos culturales que rigen la visión del mundo, ya que el sujeto nace en una sociedad constituida. Para que estos estados sean operativos deben ser por definición in- 
conscientes, aunque en ciertas circunstancias los sujetos puedan reflexionar sobre estos aspectos. Como estos principios sociales son transferidos de generación en generación, es posible hablar de regularidades, es decir, manifestaciones materiales que se producen con cierta frecuencia estadísticamente mensurable. Estos principios sociales "asociados a una clase particular de condiciones de existencia producen habitus, sistemas de disposiciones duraderas y transferibles [...] principios generadores y organizadores de prácticas y representaciones [...] objetivamente regulados y regulares sin ser el producto de la obediencia a reglas" (Bourdieu 2007 [1980]:86). Por estas razones, lograr el conocimiento práctico para la confección de enseres cerámicos habría implicado incorporar a través de un sistema de enseñanza informal los gestos técnicos y la práctica como método de aprendizaje, el cual paralelamente comunicaría valores sociales. Estas regularidades técnicas trasmitidas a través del tiempo de forma práctica conformarían "un sistema de esquemas incorporados que, constituidos en el curso de la historia colectiva, son adquiridos en el curso de la historia individual y funcionan en la práctica y para la práctica (y no para unos fines de puro conocimiento)" (Bourdieu 1988 [1979]:478).

En última instancia, entender estos posibles hábitos de producción permitirá una aproximación al conocimiento de las sociedades que estudiamos. Las comunidades que habitaron el Campo del Pucará en los primeros siglos d.C. son conocidas por las particulares formas que tienen sus configuraciones espaciales. Según Núñez Reguerio (1998), estas poblaciones poseían un patrón de asentamiento común compuesto por una aparente depresión central (patio central), un anillo sobreelevado causado por distintas estructuras monticulares (recintos) -generalmente ovaladas, ocasionalmente circulares-que lo bordean y limitan, cuyos ejes se orientan a la "depresión central". A su vez, en esta estructuración espacial se distinguen dos montículos (plataformas) ubicados generalmente en la parte occidental del anillo con orientación norte-sur de sus ejes mayores, delimitados con cuatro paredes de piedras y los restantes montículos. Al oeste de este anillo, se encuentra en forma constante un montículo (basurero) de grandes dimensiones, tanto en extensión como en altura, con abundantes restos materiales en superficie (Núñez Reguerio 1998:figuras 7, 8, 9,10, 11, 12, y 13). También, este patrón arquitectónico particular se destaca tanto por el registro arqueológico relacionado con el abandono de las estructuras habitacionales como porque no ha sido observado en otras partes del Noroeste argentino (Gordillo y Leiton 2015). Los fechados radiocarbónicos obtenidos en el área han determinado que estas ocupaciones humanas tuvieron lugar durante los primeros siglos d.C. (Angiorama 1996-98).

A lo largo de las décadas posteriores a las excavaciones, los estudios alfareros resultaron en una clasificación tipológica y estuvieron enfocados en resolver, principalmente, problemáticas referentes a la cronología relativa de las ocupaciones humanas del área. Así, a principios de la década de 1970 se establecieron tipos cerámicos en relación con la presencia o ausencia de diseños plásticos. De esta forma, se conformó un conjunto caracterizado por no tener tratamientos estéticos, pero con una amplia variabilidad granulométrica de las inclusiones entre los distritos tipos que lo integran: Alumbrera Ordinario, Caspicuchuna Ordinario, Ojo de Agua Ordinario, Alumbrera Monocromo Rojo, Alumbrera Pulido, Ciénaga Gris Liso, Ciénaga Anaranjado Liso, Condorhuasi Monocromo Rojo. Por otro lado, se configuraros dos series relacionadas con la presencia de representaciones incisas (Aconquija Inciso y sus variantes, Alumbrar Inciso, Campo del Pucará Inciso, Caspicuchuna Inciso, Ciénaga Inciso y sus variantes) o por el uso de la técnica de la pintura (Alumbrera Líneas Paralelas, Alumbrera Pintado, Alumbrera Post-cocción, Alumbrera Tricolor, Caspicuchuna Blanco sobre Ante, Caspicuchuna Negativo, Ciénaga Negro sobre Ante, Ciénaga Negro sobre Crema, Ciénaga Rojo sobre Ante, Condorhuasi Blanco sobre Rojo, Condorhuasi Polícromo y Condorhuasi Rojo sobre Ante). Estos tipos cerámicos con diseños plásticos también presentan variabilidad granulométrica entre ellos (Núñez Reguiro 1998).

A pesar de este ordenamiento, poco se conoce sobre cómo elaboraban sus vasijas las artesanas y artesanos que habitaron estos poblados Alamito. La investigación aquí planteada tiende 
a superar esta clasificación tipológica analizando las pastas cerámicas por medio del estudio petrográfico, con el objetivo de aproximarnos a las técnicas alfareras. Así, se procura poner de manifiesto las regularidades y variabilidades existentes, a partir de las cuales se podrá contribuir al conocimiento de los antiguos modos de hacer vasijas que poseyeron las alfareras y alfareros de las antiguas sociedades que habitaron estas aldeas en los primeros siglos d.C., es decir, durante el Período Formativo.

Finalmente, este trabajo propone, además, poner en valor una antigua colección arqueológica depositada en el Museo de Antropología de Córdoba, en tal sentido este tipo de estudios contribuyen a una de las funciones que ha sido encomendada a los museos como "instituciones que rescatan, investigan y valorizan [...] la realidad pasada y presente" (Pérez Gollán y Dujovne, 1995:120). Es decir, que la investigación también pretende revalorizar los fondos museísticos a través del estudio de una colección vapuleada por los avatares político-sociales producidos por los golpes militares de 1966 y 1976 en la República Argentina (Núñez Regueiro 1998: xxix).

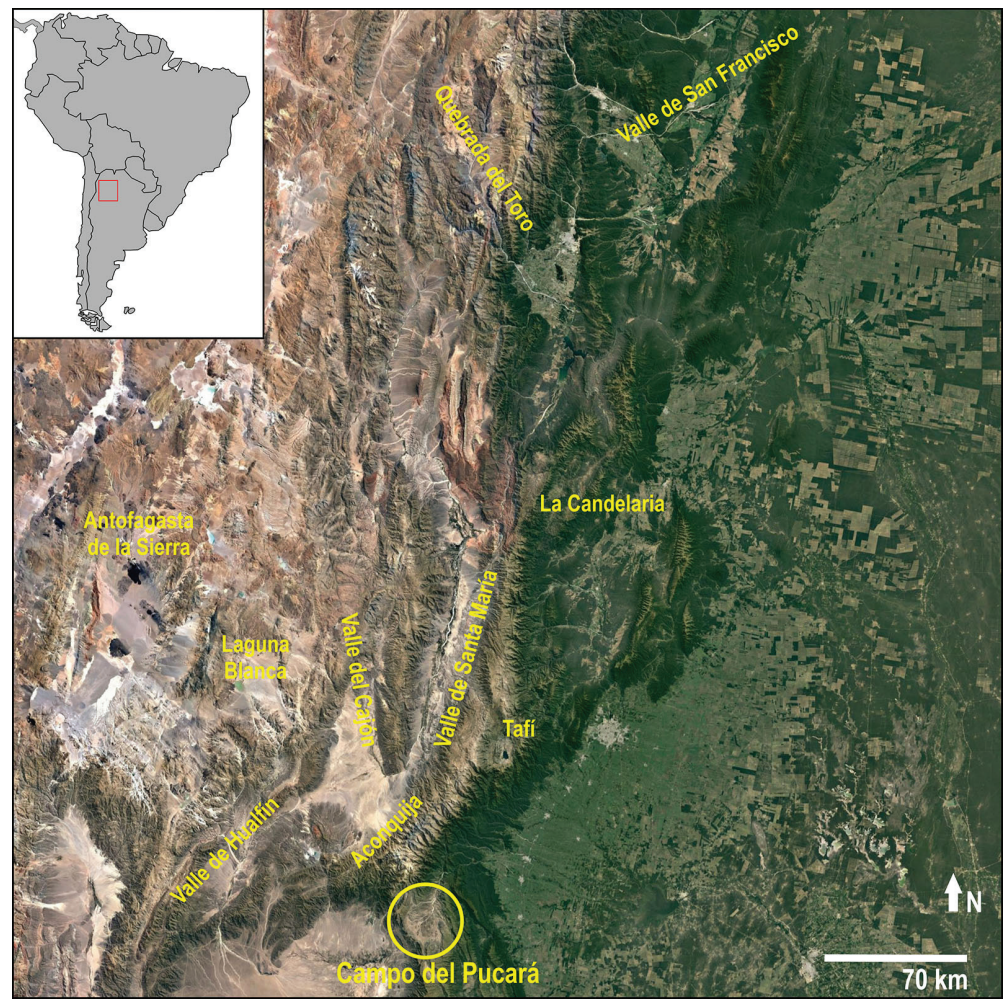

Figura 1. Ubicación de Campo del Pucará, área de asentamiento de los sitios Alamito, y de otras regiones mencionadas

\section{MUESTRA Y METODOLOGÍA}

La selección de la muestra cerámica estuvo orientada a comprender la producción del universo estilístico de la alfarería de los sitios Alamito. Ésta se conoce a partir de las distintas excavaciones realizadas por Víctor Núñez Regueiro entre los años 1957 y 1966. Actualmente, parte de esas colecciones se encuentran depositadas en el Museo de Antropología de la Universidad Nacional de Córdoba. De ese acervo, se analizaron 1801 fragmentos, entre los que se seleccionaron los 55 
que conforman la muestra analizada (tabla 1). Con el objetivo de testear la variabilidad estilístico-morfológica de estos tiestos, se atendieron variables como la procedencia, los tipos cerámicos, el número mínimo de vasijas (NMV) y las familias de fragmentos. Las distintas procedencias se registraron a partir de la información disponible en el depósito del Museo de Antropología. La clasificación tipológica se alcanzó a través de los lineamientos establecidos por Núñez Regueiro (1998) para el área de Campo del Pucará y una actualización propuesta para el área de la falda occidental del Aconquija (Bugliani 2008; Pereyra Domingorena 2012, 2015). La clasificación morfológica de las vasijas reconstruidas siguió los criterios morfométricos especificados por Balfet y otros (1983). Esta tipología clasificatoria se adaptó a las características propias de la muestra y variantes en el interior del tipo morfológico, teniendo en cuenta consideraciones en relación con el tamaño, la simetría, la presencia de asas y otras características que permitieron determinar diferenciaciones (Bugliani 2008). Además, se utilizó el concepto de familia de fragmentos tal como lo definiera Orton y otros (1997). Es así que se pudo constituir una muestra que representa los tipos cerámicos establecidos en el área y se tuvo en cuenta que los fragmentos perteneciesen a distintos recipientes, para asegurar la expresión de la posible variabilidad petrográfica de las pastas cerámicas.

De esta manera los 55 fragmentos seleccionados corresponden a tipos cerámicos como Condorhuasi Polícromo, Río Diablo, Ciénaga Pintado y otros estilos cerámicos del Período Formativo (tabla 1). La figura 2 ilustra algunos de los tiestos que conforman la muestra: a) M-01: Gris pulido Inciso (pertenece a un fragmento de un jarro o jarra de estilo Río Diablo, posiblemente se corresponda a una variante del tipo Ciénaga Inciso; b) M-06: Condorhuasi (posiblemente Condorhuasi Rojo sobre Blanco); c) M-15: Ante Alisado Inciso (¿Alpatauca?_Alumbrera Inciso?); d) M-22: Gris Pulido Inciso; e) M-24: Gris Pulido Inciso; f) M-29: Ordinario (fragmento de una olla con tizne en la superficie externa); g) M-38: Ciénaga Negro sobre Crema; h) M-45: Alumbrera Líneas Paralelas; i) M-46: Alumbrera Tricolor. Además, se pudieron reconocer 9 recipientes que

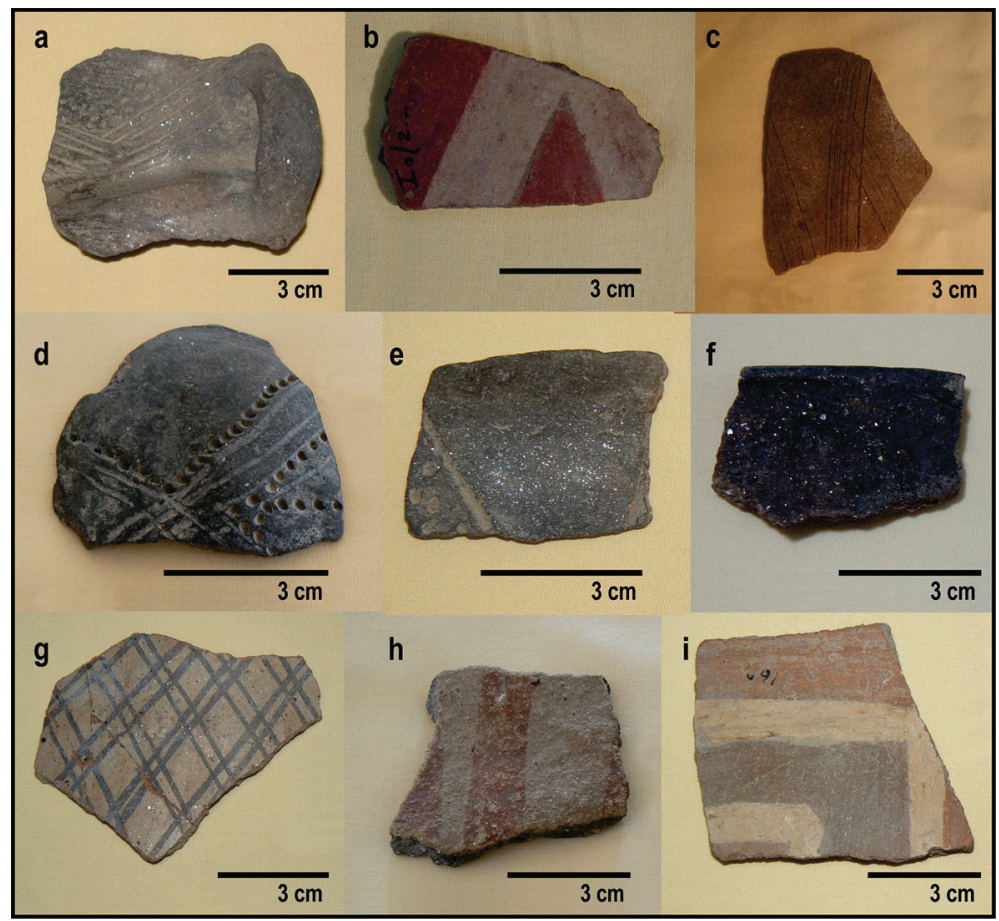

Figura 2. Ejemplos de algunos tipos cerámicos hallados en el área 
se corresponden a distintas morfologías. En las figuras 3 y 4 se pueden observar las siluetas de las piezas reconstruidas: 3a) olla ordinaria alisada (M-54); 3b) jarra Gris Pulida Incisa (M-17); 3c) olla Condorhuasi Blanco sobre Rojo (M-47); 3d) jarra Alumbrera Líneas Paralelas (M-43); 4a) cuenco con diseños sólidos en pintura negra sobre el fondo beige de la pasta (Ciénaga Negro sobre Ante) (M-10); 4b) cuenco con punto angular y pintura negra sobre el fondo beige de la pasta (Ciénaga Negro sobre Ante) (M-37); 4c) jarrito Condorhuasi Polícromo (M-41); 4d) escudilla gris pulida incisa (M-53); 4e) jarro gris-negro pulido inciso (M-49). Otros fragmentos no han servido para determinar siluetas, pero corresponderían a recipientes cerrados -quizá ollas- con restos de hollín en sus superficies externas.

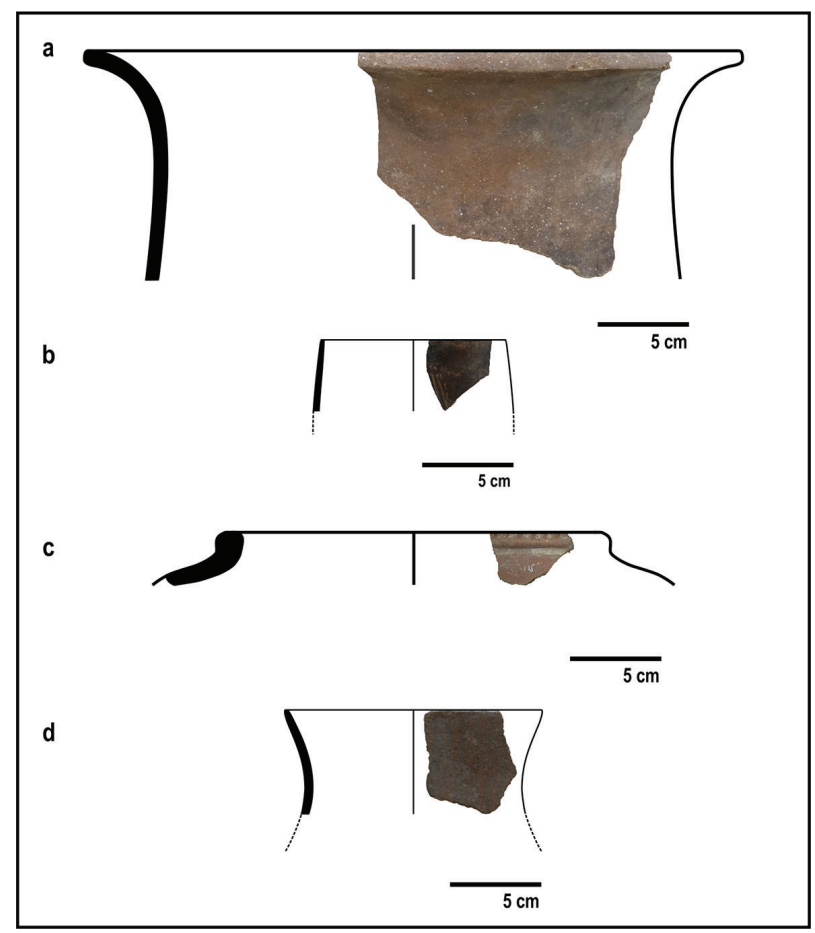

Figura 3. Siluetas de recipientes parcialmente reconstruidos registrados en la muestra analizada

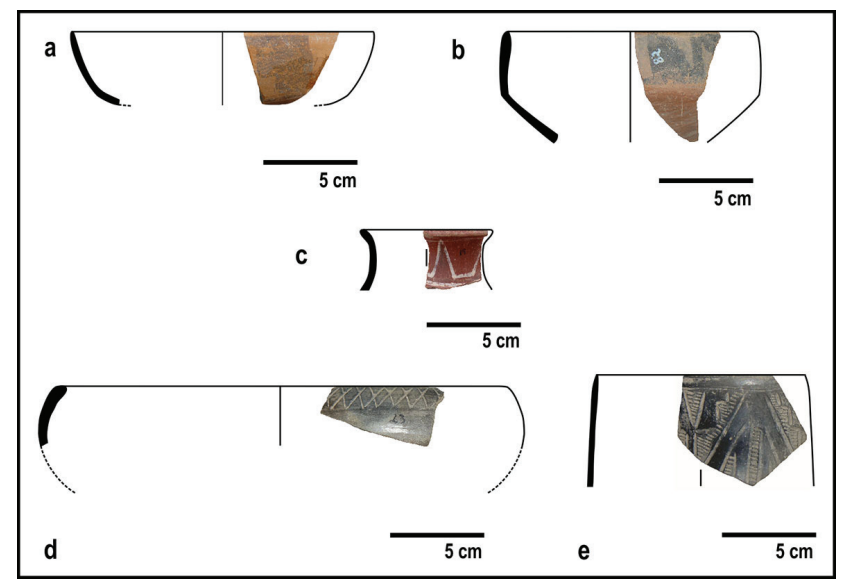

Figura 4. Siluetas de recipientes parcialmente reconstruidos registrados en la muestra analizada 
De cada uno de los 55 tiestos se confeccionó una sección delgada de la pasta (30 بm de espesor) para su análisis en un microscopio de polarización Leica DM EP con accesorio fotográfico y platina graduada para point counting. Para cada corte delgado se obtuvo una caracterización cuantitativa y composicional de las pastas cerámicas.

El procedimiento instrumentado en este estudio incluyó cuatro fases. En primer lugar, se clasificaron las estructuras de los fondos de pastas (Courtois 1976) y su análisis textural (Middleton et al. 1991). La segunda fase consistió en la determinación mineralógica ${ }^{1}$ de las inclusiones mayores a $15 \mu \mathrm{m}$ (granulometría limo medio). Estas se clasificaron en cristaloclastos, es decir, fragmentos de cristales o cristales de minerales individuales y fragmentos de rocas -litoclastos-, los cuales pueden tener un origen ígneo (volcánico o plutónico), sedimentario o metamórfico; además, se observaron otros elementos como minerales opacos, vidrio volcánico, nódulos de arcilla y tiesto molido. Las formas y hábitos fueron clasificados de acuerdo a las cartillas utilizadas en sedimentología y para los tamaños de las inclusiones se utilizó la escala de clasificación granulométrica de Wentworth (ambas tomadas de Adams y otros 1997 [1984]). En la tercera fase, se reconocieron y clasificaron las cavidades (poros y oquedades) presentes en las pastas. Se describió su abundancia, tamaños y formas. La cuarta, y última fase, consistió en el cálculo porcentual de los componentes que integran la pasta (matriz, inclusiones y cavidades). Para ello se efectuó un conteo mínimo de 300 puntos (point counting) por corte delgado, aplicándose el método de intercepción múltiple (multiple interseption). Cada sección delgada fue "barrida" de izquierda a derecha (a intervalos de $1 \mathrm{~mm}$ ), registrándose -en la intersección de los hilos del ocular y a una magnificación de 100x- la matriz, el tipo de inclusión no plástica o la cavidad presente. Durante esta etapa además se examinaron las formas y tamaños tanto de las inclusiones no plásticas como de las cavidades, utilizando para ello la escala micrométrica del microscopio y considerando su eje máximo. El conteo se realizó en forma manual mediante escala graduada agregada como accesorio a la platina giratoria del microscopio de polarización. En suma, el procedimiento implementado permitió caracterizar la pasta cerámica mediante la descripción de las estructuras de fondo de la matriz arcillosa y la cuantificación e identificación tanto de los materiales no plásticos como de las cavidades.

Se ensayó de modo exploratorio un ordenamiento de la muestra mediante el Análisis de Conglomerados (cluster analysis) provisto por el programa PAST versión 2.17 (Hammer et al. 2001). Para dicha prueba estadística se utilizaron los valores en porcentaje obtenidos mediante point counting. Para cada sección delgada, se consideraron 13 variables cuantitativas: matriz $(\mathrm{Mz})$, cavidades $(\mathrm{Cv})$, cuarzo $(\mathrm{Qz})$, feldespato potásico $(\mathrm{FK})$, plagioclasas $(\mathrm{Pg})$, biotita $(\mathrm{Bi})$, muscovita $(\mathrm{Mu})$, anfíboles y/o piroxenos $(\mathrm{A} / \mathrm{P})$, minerales opacos $(\mathrm{Op})$; litoclastos graníticos o granodioríticos (LG), litoclastos de esquisto (Eq), litoclastos volcánicos (LV), vidrio volcánico (VV) (tabla 2). Otras variables tales como microclino, granate, turmalina, arenisca, carbonatos y tiesto molido no fueron consideradas por tener una presencia esporádica e ínfima en algunas muestras. El análisis de conglomerados se realizó utilizando el algoritmo de pares agrupados con medida de distancia euclidiana (Shennan 1992 [1988]). De esta prueba, se obtuvo un coeficiente de correlación cofenética de 0,6663, siendo el fenograma resultante una representación poco confiable de la matriz de distancia (figura 5). Ante este resultado inesperado, se realizó un Análisis de Componentes Principales (ACP) con las mismas variables utilizadas para el análisis de conglomerados y con el programa estadístico antes mencionado. El resultado de este segundo ensayo presentó una distribución de las muestras que no permite segregar cabalmente agrupaciones en el conjunto cerámico estudiado (figura 6). Por lo tanto, las asociaciones discriminadas en ambas pruebas no fueron concluyentes para la conformación de los tipos de pastas, aunque esta metodología estadística sí ha servido en otras investigaciones (Cremonte 1996; Pereyra Domingorena 2012, 2015; Puente 2012; Cremonte et. al. 2016; De Feo y Pereyra Domingorena 2018; Pereyra Domingorena y Bugliani 2019; Gasparoti y Escola 2020). Se piensa que esta situación 
se debe a la homogeneidad de los materiales no plásticos - principalmente por los componentes cuarzo y litoclastos graníticos- que forman parte de las pastas analizadas y que, en el caso del análisis de componentes principales, representan las variables de mayor peso en su conformación.

Aunque los resultados de las pruebas estadísticas no hayan agrupado a la totalidad o a una gran mayoría de las muestras, los datos han permitido concebir un cimiento para guiar la configuración de un ordenamiento de los ejemplares examinados. Junto con esta base, los resultados obtenidos en los estudios previos antes mencionados de áreas vecinas del Noroeste argentino, y la combinación tanto de los atributos cualitativos registrados en la muestra analizada (estructura de fondo, formas y tamaño de las inclusiones) como de rasgos cuantitativos (porcentaje de matriz, cavidades y tipos de inclusiones) permitieron clasificar las pastas en gruesas, medias y finas -según la combinación del tamaño de las inclusiones y su abundancia- y configurar seis agrupamientos tentativos en el conjunto estudiado.

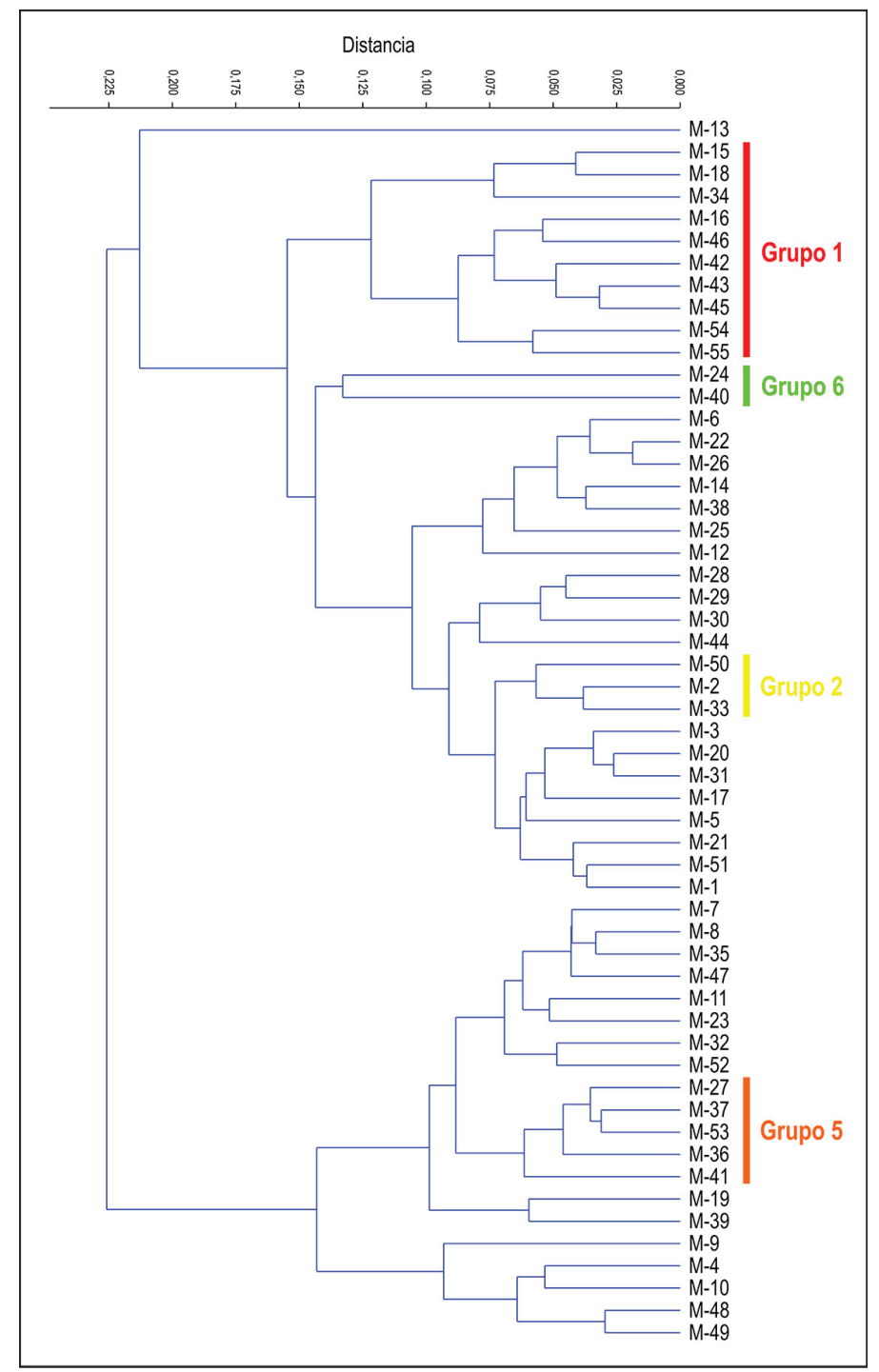

Figura 5. Dendograma y los ejemplares asignados a los grupos de pasta de forma estadística 


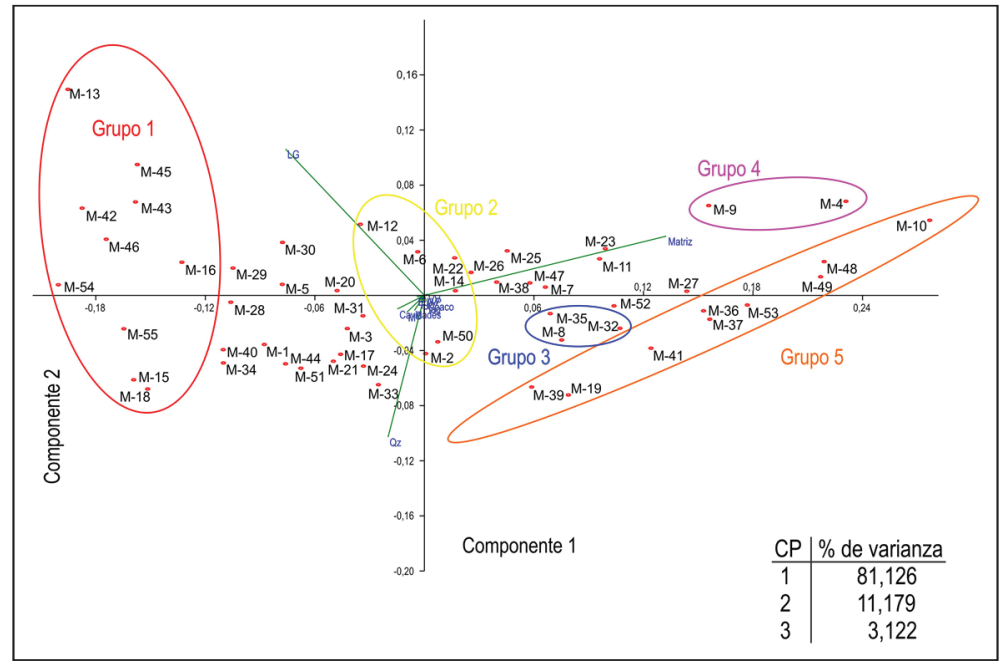

Figura 6. Gráfico del Análisis de Componentes Principales y los ejemplares asignados a los grupos de pasta de forma estadística

\section{UNA CLASIFICACIÓN DE LAS PASTAS CERÁMICAS}

Los seis grupos de pastas, que a continuación describiremos, corresponden a un ordenamiento establecido por las características cualitativas y cuantitativas registradas en cada corte delgado, por lo que es una clasificación independiente de otras aplicadas a la cerámica, como ser la estilística (Culbert y Rands 2007).

El Grupo 1 (M-1, 3, 5, 13, 15, 16, 18, 20, 28, 34, 42, 43, 44, 45, 46, 54, 55 [tablas 1 y 2 y figuras 5, 6 y 7]) está integrado por pastas gruesas que presentan como material no plástico: cuarzo (7\%-18,5\%), feldespato potásico $(0,5 \%-5,5 \%)$, plagioclasas $(0,2 \%-2 \%)$, microclino $(\leq 0,8 \%)$, biotita $(0,2 \%-5,3 \%)$, muscovita $(0,6 \%-5 \%)$, anfíboles-piroxenos $(\leq 0,4 \%)$, litoclastos graníticos $(9 \%-30 \%)$, esquisto $(\leq 3,5 \%)$, litoclastos volcánico $(\leq 1,1 \%)$, trizas volcánicas $(\leq 0,2 \%)$ y minerales opacos $(\leq 1,3 \%)$. Las inclusiones oscilan entre $35 \%$ y $45 \%$ del total de la pasta y principalmente corresponden a agregado de una arena no seleccionada, cuya granulometría oscila entre limo medio y canto pequeño $(15 \mu \mathrm{m}-4000 \mu \mathrm{m})$. Las texturas de estas pastas se destacan por presentar estructura de fondo pseudolepidoblástica, algo microgranosa. La matriz ocupa entre $47 \%$ y $60 \%$ del total de la pasta. Las inclusiones tienen formas subangulares y subredondeadas. Las cavidades representan entre $3 \%$ y $12 \%$. La coloración castaña del fondo de pasta estaría indicando el carácter oxidante de la cocción.

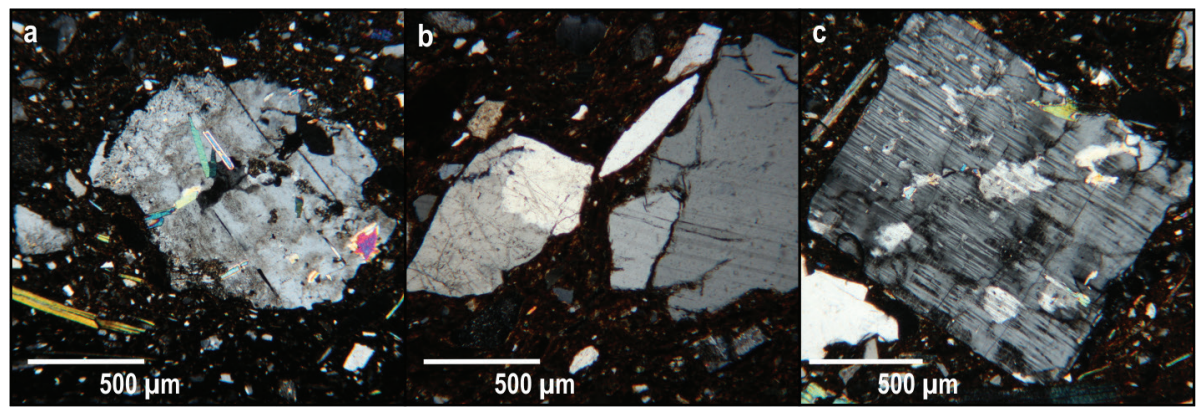

Figura 7. Fotomicrografías de pastas del Grupo 1: a) M-1; b) M-46; c) M-54 
Relaciones de la Sociedad Argentina de Antropología 46 (2), julio-diciembre 2021: 503-525

Tabla 1. Muestras cerámicas analizadas, tipos cerámicos asignados, procedencias de los fragmentos y grupos de pasta asociados

\begin{tabular}{|c|c|c|c|}
\hline Corte & Tipo Cerámico & Procedencia & GP \\
\hline M-01 & $\begin{array}{l}\text { Gris Pulido Inciso (estilo Río Diablo, } \\
\text { posiblemente una variante del tipo Ciénaga } \\
\text { Inciso) }\end{array}$ & $\begin{array}{l}\text { Sitio B-0. Montículo mayor. Pozo de } \\
\text { sondeo. Capa } 10\end{array}$ & 1 \\
\hline M-02 & Condorhuasi Blanco sobre Rojo & $\begin{array}{l}\text { Posible capa } 1 \text { del montículo mayor del } \\
\text { sitio I-0 }\end{array}$ & 2 \\
\hline M-03 & $\begin{array}{l}\text { Ordinario Alisado con modelado inciso (no } \\
\text { ha sido posible adscribir a un tipo cerámico } \\
\text { de Alamito) }\end{array}$ & $\begin{array}{l}\text { Posible capa } 14 \text { del montículo mayor del } \\
\text { sitio C-1 }\end{array}$ & 1 \\
\hline M-04 & $\begin{array}{l}\text { Gris Pulido (no ha sido posible adscribir a } \\
\text { un tipo cerámico de Alamito) }\end{array}$ & $\begin{array}{l}\text { Posible capa } 8 \text { del montículo mayor del } \\
\text { sitio C-0 }\end{array}$ & 4 \\
\hline M-05 & $\begin{array}{l}\text { Ordinario Alisado Pared delgada (no ha } \\
\text { sido posible adscribir a un tipo cerámico de } \\
\text { Alamito) }\end{array}$ & $\begin{array}{l}\text { Posible capa } 6 \text { del montículo mayor del } \\
\text { sitio C- } 0\end{array}$ & 1 \\
\hline M-06 & $\begin{array}{l}\text { Condorhuasi (¿Condorhuasi Blanco sobre } \\
\text { rojo?) }\end{array}$ & $\begin{array}{l}\text { Posible capa } 2 \text { del montículo mayor del } \\
\text { sitio I-0 }\end{array}$ & 2 \\
\hline M-07 & $\begin{array}{l}\text { Ordinario Alisado Pared delgada (¿Ojo de } \\
\text { Agua Ordinario?) }\end{array}$ & $\begin{array}{l}\text { Posible capa } 9 \text { del montículo mayor del } \\
\text { sitio M-1 }\end{array}$ & 4 \\
\hline M-08 & $\begin{array}{l}\text { Ordinario Alisado (¿Ojo de Agua } \\
\text { Ordinario?) }\end{array}$ & $\begin{array}{l}\text { Posible capa } 4 \text { del montículo mayor del } \\
\text { sitio C- } 0\end{array}$ & 3 \\
\hline M-09 & Ciénaga Negro sobre Crema & $\begin{array}{l}\text { Posible capa } 2 \text { del montículo mayor del } \\
\text { sitio K-0 }\end{array}$ & 4 \\
\hline M-10 & Ciénaga Negro sobre Ante & $\begin{array}{l}\text { Posible capa } 2 \text { del montículo mayor del } \\
\text { sitio K-0 }\end{array}$ & 5 \\
\hline M-11 & $\begin{array}{l}\text { Baño Blanco Pulido Inciso (¿variante del } \\
\text { tipo Ciénaga Inciso?) }\end{array}$ & $\begin{array}{l}\text { Posible capa } 4 \text { del montículo mayor del } \\
\text { sitio I-0 }\end{array}$ & 4 \\
\hline M-12 & $\begin{array}{l}\text { Ordinario Alisado Pintado (Alumbrera } \\
\text { Pintado) }\end{array}$ & $\begin{array}{l}\text { Sitio B-0. Montículo mayor. Pozo de } \\
\text { sondeo. Capa } 11\end{array}$ & 2 \\
\hline M-13 & $\begin{array}{l}\text { Ordinario Alisado (no ha sido posible } \\
\text { adscribir a un tipo cerámico de Alamito) }\end{array}$ & $\begin{array}{l}\text { Sitio B-0. Montículo mayor. Pozo de } \\
\text { sondeo. Capa } 11\end{array}$ & 1 \\
\hline M-14 & Ordinario Pulido (¿Alumbrera Pulido?) & $\begin{array}{l}\text { Sitio B-0. Montículo mayor. Pozo de } \\
\text { sondeo. Capa } 11\end{array}$ & 2 \\
\hline M-15 & $\begin{array}{l}\text { Ante Alisado Inciso (¿Alpatauca? } \\
\text { ¿Alumbrera Inciso?) }\end{array}$ & $\begin{array}{l}\text { Sitio B-0. Montículo mayor. Pozo de } \\
\text { sondeo. Capa } 11\end{array}$ & 1 \\
\hline M-16 & $\begin{array}{l}\text { Blanco sobre Rojo Alisado e Inciso (no ha } \\
\text { sido posible adscribir a un tipo cerámico de } \\
\text { Alamito) }\end{array}$ & $\begin{array}{l}\text { Sitio B-0. Montículo mayor. Pozo de } \\
\text { sondeo. Capa } 11\end{array}$ & 1 \\
\hline M-17 & $\begin{array}{l}\text { Gris Pulido Inciso (posiblemente una } \\
\text { variante del tipo Aconquija Inciso) }\end{array}$ & $\begin{array}{l}\text { Sitio B-0. Montículo mayor. Pozo de } \\
\text { sondeo. Capa } 12\end{array}$ & 2 \\
\hline M-18 & $\begin{array}{l}\text { Ordinario Alisado Inciso y Pintado (no ha } \\
\text { sido posible adscribir a un tipo cerámico de } \\
\text { Alamito) }\end{array}$ & $\begin{array}{l}\text { Sitio B-0. Montículo mayor. Pozo de } \\
\text { sondeo. Capa } 12\end{array}$ & 1 \\
\hline M-19 & $\begin{array}{l}\text { Gris Pulido Inciso (¿Campo del Pucará } \\
\text { Inciso?) }\end{array}$ & $\begin{array}{l}\text { Sitio B-0. Montículo mayor. Pozo de } \\
\text { sondeo. Capa } 12\end{array}$ & 5 \\
\hline M-20 & $\begin{array}{l}\text { Ordinario Alisado Inciso Pared delgada (no } \\
\text { ha sido posible adscribir a un tipo cerámico } \\
\text { de Alamito) }\end{array}$ & $\begin{array}{l}\text { Sitio B-0. Montículo mayor. Pozo de } \\
\text { sondeo. Capa } 10\end{array}$ & 1 \\
\hline
\end{tabular}


(Tabla 1. Continuación)

\begin{tabular}{|c|c|c|c|}
\hline Corte & Tipo Cerámico & Procedencia & GP \\
\hline M-21 & Condorhuasi Rojo sobre Ante & $\begin{array}{l}\text { Sitio B-0. Montículo mayor. Pozo de } \\
\text { sondeo. Capa } 09\end{array}$ & 2 \\
\hline M-22 & $\begin{array}{l}\text { Gris Pulido Inciso (no ha sido posible } \\
\text { adscribir a un tipo cerámico de Alamito) }\end{array}$ & $\begin{array}{l}\text { Sitio B-0. Montículo mayor. Pozo de } \\
\text { sondeo. Capa } 09\end{array}$ & 2 \\
\hline M-23 & $\begin{array}{l}\text { Condorhuasi (puede corresponder a varios } \\
\text { tipos dentro de esta cerámica) }\end{array}$ & $\begin{array}{l}\text { Sitio B-0. Montículo mayor. Pozo de } \\
\text { sondeo. Capa } 13\end{array}$ & 3 \\
\hline M-24 & $\begin{array}{l}\text { Gris Pulido Inciso (no ha sido posible } \\
\text { adscribir a un tipo cerámico de Alamito) }\end{array}$ & $\begin{array}{l}\text { Sitio B-0. Montículo mayor. Pozo de } \\
\text { sondeo. Capa } 13\end{array}$ & 6 \\
\hline M-25 & $\begin{array}{l}\text { Ordinario Alisado (no ha sido posible } \\
\text { adscribir a un tipo cerámico de Alamito) }\end{array}$ & $\begin{array}{l}\text { Sitio B-0. Montículo mayor. Pozo de } \\
\text { sondeo. Capa } 04\end{array}$ & 3 \\
\hline M-26 & $\begin{array}{l}\text { Ordinario Pintado (¿Alumbrera } \\
\text { Monocromo Rojo?) }\end{array}$ & U. H1. M1. R1/7 & 3 \\
\hline M-27 & $\begin{array}{l}\text { Ordinario Pintado (¿Alumbrera } \\
\text { Monocromo Rojo?) }\end{array}$ & U. H1. M1. R1/7 & 5 \\
\hline M-28 & $\begin{array}{l}\text { Ordinario Pintado (¿Alumbrera } \\
\text { Monocromo Rojo?) }\end{array}$ & U. H1. M1. R1/7 & 1 \\
\hline M-29 & $\begin{array}{l}\text { Ordinario Alisado (no ha sido posible } \\
\text { adscribir a un tipo cerámico de Alamito) }\end{array}$ & U. H1. M1. R1/7 & 3 \\
\hline M-30 & $\begin{array}{l}\text { Ordinario Alisado (no ha sido posible } \\
\text { adscribir a un tipo cerámico de Alamito) }\end{array}$ & U. H1. M1. R1/7 & 3 \\
\hline M-31 & $\begin{array}{l}\text { Ordinario Alisado (no ha sido posible } \\
\text { adscribir a un tipo cerámico de Alamito) }\end{array}$ & U. H1. M1. R1/7 & 3 \\
\hline M-32 & $\begin{array}{l}\text { Ordinario Alisado (no ha sido posible } \\
\text { adscribir a un tipo cerámico de Alamito) }\end{array}$ & U. H1. M1. R1/7 & 3 \\
\hline M-33 & Ciénaga Rojo sobre Ante & U. H1. M1. R1/7 & 2 \\
\hline M-34 & $\begin{array}{l}\text { Ordinario Alisado (no ha sido posible } \\
\text { adscribir a un tipo cerámico de Alamito) }\end{array}$ & U. H1. M1. R1/7 & 1 \\
\hline M-35 & $\begin{array}{l}\text { Ordinario Alisado (no ha sido posible } \\
\text { adscribir a un tipo cerámico de Alamito). }\end{array}$ & U. H1. M1. R1/7 & 3 \\
\hline M-36 & $\begin{array}{l}\text { Gris Pulido Inciso (posiblemente una } \\
\text { variante del tipo Ciénaga Inciso) }\end{array}$ & U. D1. M2. Piso inferior & 5 \\
\hline M-37 & Ciénaga Negro sobre Ante & U. D. M4. H1 & 5 \\
\hline M-38 & Ciénaga Negro sobre Crema & U. D. M4. H1 & 4 \\
\hline M-39 & $\begin{array}{l}\text { Gris Pulido Inciso (posiblemente una } \\
\text { variante del tipo Ciénaga Inciso) }\end{array}$ & U. D. M4. H1 & 5 \\
\hline M-40 & $\begin{array}{l}\text { Ordinario Alisado (no ha sido posible } \\
\text { adscribir a un tipo cerámico de Alamito) }\end{array}$ & U. D. M4. H1 & 6 \\
\hline M-41 & Condorhuasi Polícromo & U. D. M 6/R3 & 5 \\
\hline M-42 & Alumbrera Líneas Paralelas & $\begin{array}{l}\text { Sitio B-0. Montículo mayor. Pozo de } \\
\text { sondeo. Capa } 10\end{array}$ & 1 \\
\hline M-43 & Alumbrera Líneas Paralelas & $\begin{array}{l}\text { Sitio B-0. Montículo mayor. Pozo de } \\
\text { sondeo. Capa } 10\end{array}$ & 1 \\
\hline M-44 & Alumbrera Post-cocción & $\begin{array}{l}\text { Sitio B-0. Montículo mayor. Pozo de } \\
\text { sondeo. Capa } 10\end{array}$ & 1 \\
\hline M-45 & Alumbrera Líneas Paralelas & $\begin{array}{l}\text { Sitio B-0. Montículo mayor. Pozo de } \\
\text { sondeo. Capa } 10\end{array}$ & 1 \\
\hline
\end{tabular}


Relaciones de la Sociedad Argentina de Antropología 46 (2), julio-diciembre 2021: 503-525

(Tabla 1. Continuación)

\begin{tabular}{|c|c|c|c|}
\hline Corte & Tipo Cerámico & Procedencia & GP \\
\hline M-46 & Alumbrera Tricolor & Mesada El Arbolito & 1 \\
\hline M-47 & Condorhuasi Blanco sobre Rojo & Mesada El Arbolito & 3 \\
\hline M-48 & $\begin{array}{l}\text { Gris Pulido Grabado (no ha sido posible } \\
\text { adscribir a un tipo cerámico de Alamito) }\end{array}$ & Mesada El Arbolito & 5 \\
\hline M-49 & $\begin{array}{l}\text { Gris-Negro Pulido Inciso (posiblemente } \\
\text { una variante del tipo Ciénaga Inciso) }\end{array}$ & Mesada El Arbolito & 5 \\
\hline M-50 & $\begin{array}{l}\text { Gris Pulido Inciso (posiblemente una } \\
\text { variante del tipo Aconquija Inciso) }\end{array}$ & Mesada El Arbolito & 2 \\
\hline M-51 & Ciénaga Negro sobre Ante & Mesada El Arbolito & 4 \\
\hline M-52 & $\begin{array}{l}\text { Negro Pulido Inciso (no ha sido posible } \\
\text { adscribir a un tipo cerámico de Alamito) }\end{array}$ & Mesada El Arbolito & 5 \\
\hline M-53 & $\begin{array}{l}\text { Gris Pulido Inciso (no ha sido posible } \\
\text { adscribir a un tipo cerámico de Alamito) }\end{array}$ & Mesada El Arbolito & 5 \\
\hline M-54 & $\begin{array}{l}\text { Ordinario Alisado (no ha sido posible } \\
\text { adscribir a un tipo cerámico de Alamito) }\end{array}$ & Alamito & 1 \\
\hline M-55 & $\begin{array}{l}\text { Ordinario Alisado (no ha sido posible } \\
\text { adscribir a un tipo cerámico de Alamito) }\end{array}$ & Alamito & 1 \\
\hline
\end{tabular}

Referencias: GP: Grupo de pasta

El Grupo 2 (M-2, 6, 12, 14, 17, 21, 22, 33, 50 [tablas 1 y 2 y figuras 5, 6 y 8]) está conformado por pastas gruesas de densidad moderada que presentan como material no plástico: cuarzo $(9,5 \%-20 \%)$, feldespato potásico $(0,6 \%-3,6 \%)$, plagioclasas $(\leq 0,9 \%)$, microclino $(\leq 0,4 \%)$, biotita $(0,7 \%-3,6 \%)$, muscovita $(0,6 \%-2,7 \%)$, anfíboles-piroxenos $(\leq 0,8 \%)$, litoclastos graníticos $(6,7 \%$ $4,6 \%)$, esquisto $(\leq 0,9 \%)$, litoclastos volcánico $(\leq 0,3 \%)$, trizas volcánicas $(\leq 0,3 \%)$, minerales opacos $(\leq 1,3 \%)$. Las inclusiones oscilan entre $28 \%$ y $35 \%$ del total de la pasta y principalmente corresponden a agregado de una arena no seleccionada, cuya granulometría oscila entre limo medio y arena muy gruesa $(15 \mu \mathrm{m}-2000 \mu \mathrm{m})$. Las texturas de estas pastas se destacan por presentar estructura de fondo pseudolepidoblástica, algo microgranosa. La matriz ocupa entre 59\% y $67 \%$ del total de la pasta. Las inclusiones tienen formas subangulares y subredondeadas. Las cavidades representan entre $3 \%$ y $12 \%$. La coloración castaña del fondo de pasta estaría indicando el carácter oxidante de la cocción.

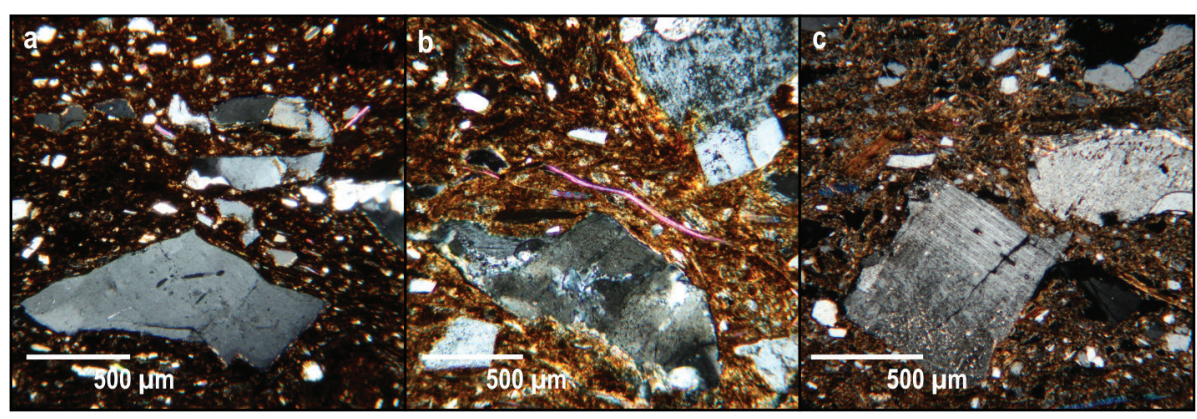

Figura 8. Fotomicrografias de pastas del Grupo 2: a) M-6; b) M-12; c) M-22 


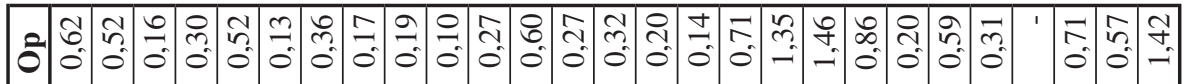

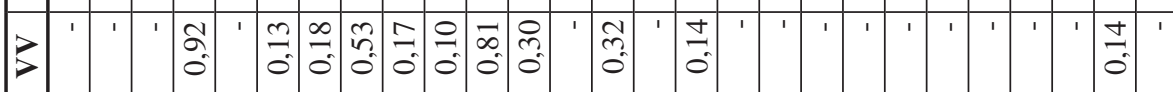

\begin{tabular}{|c|c|c|c|c|c|c|c|c|c|c|c|c|c|c|c|c|c|c|c|c|c|c|c|c|c|}
\hline Z & ' & ' & $\begin{array}{l}0 \\
0 \\
0\end{array}$ & $\begin{array}{l}\sigma \\
\delta \\
\delta\end{array}$ & ' & ' & ' & $\frac{亏}{0}$ & I & ' & $\begin{array}{l}0 \\
+ \\
i \\
\text { in }\end{array}$ & $\frac{n}{0}$ & ' & $\begin{array}{l}0 \\
0 \\
0\end{array}$ & సి & & $\mid \begin{array}{l}\infty \\
0 \\
-1\end{array}$ & $\begin{array}{c}\infty \\
n \\
0 \\
0\end{array}$ & $\begin{array}{c}\overrightarrow{\mathrm{N}} \\
\hat{0}\end{array}$ & 1 & ' & 1 & ' & 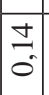 & $\bar{m}$ \\
\hline$z$ & 1 & 1 & & I & 1 & 1 & 1 & 1 & 1 & I & ' & ' & 1 & 1 & & 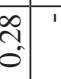 & 1 & ' & 1 & ' & ' & ' & ' & ' & \\
\hline in & & & $\begin{array}{l}2 \\
0 \\
0\end{array}$ & & \begin{tabular}{l|l}
0 \\
rn \\
$i$
\end{tabular} & $\begin{array}{l}0 \\
\hat{\sigma}\end{array}$ & ' & 1 & 1 & & 1 & ' & ' & $\begin{array}{c}0 \\
-0 \\
0\end{array}$ & & 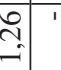 & $\left|\begin{array}{l}\infty \\
\stackrel{0}{-1}\end{array}\right|$ & & $\stackrel{m}{+}$ & ' & & & $\vec{\sigma}$ & $\left|\begin{array}{|c}\infty \\
\text { N } \\
0\end{array}\right|$ & के \\
\hline
\end{tabular}

$\mathbf{N}$ :

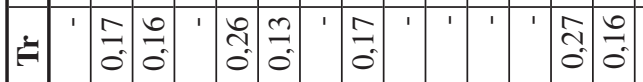

\begin{tabular}{|c|c|c|c|c|c|c|c|c|c|c|c|c|c|c|c|c|c|c|c|c|c|c|c|c|c|c|c|}
\hline 5 & $\stackrel{f}{f}$ & $\begin{array}{l}\hat{0} \\
0 \\
0\end{array}$ & $\begin{array}{l}\bar{Z} \\
\mathrm{i}\end{array}$ & & & $\mid \begin{array}{l}0 \\
0 \\
0\end{array}$ & $\begin{array}{l}0 \\
f \\
- \\
-1\end{array}$ & $\stackrel{\mathcal{I}}{=}$ & $\mid \begin{array}{c}\infty \\
\vdots \\
0\end{array}$ & 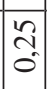 & $\left|\begin{array}{c}\hat{\lambda} \\
0\end{array}\right|$ & $\mid \begin{array}{l}0 \\
0 \\
i\end{array}$ & $\hat{n}$ & $\begin{array}{l}\mathbb{J} \\
0 \\
0\end{array}$ & $\begin{array}{l}\overline{6} \\
0\end{array}$ & $\mid \begin{array}{l}\infty \\
0 \\
0\end{array}$ & 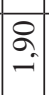 & $\begin{array}{l}0 \\
\text { ii }\end{array}$ & $\begin{array}{l}2 \\
= \\
=\end{array}$ & $\begin{array}{c}0 \\
\text { लि. } \\
-1\end{array}$ & $\underline{0}$ & $\hat{\sigma}$ & 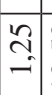 & $\begin{array}{l}0 \\
\text { ก. } \\
\text { cin }\end{array}$ & & $\begin{array}{l}\infty \\
n \\
\sim \\
- \\
-\end{array}$ & \\
\hline$\oplus$ & $\begin{array}{c}0 \\
0 \\
-1\end{array}$ & 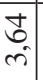 & $\begin{array}{c}2 \\
\vec{n}\end{array}$ & \begin{tabular}{|c}
0 \\
m. \\
0
\end{tabular} & $\begin{array}{l}8 \\
i\end{array}$ & $\stackrel{2}{=}$ & $\begin{array}{l}0 \\
2 \\
0 \\
0\end{array}$ & $\stackrel{8}{-}$ & $\overrightarrow{0}$ & $\begin{array}{l}0 \\
0 \\
0\end{array}$ & $\left|\begin{array}{c}+ \\
n \\
0 \\
0\end{array}\right|$ & $\begin{array}{l}n \\
0 \\
0\end{array}$ & $\mid \begin{array}{c}\hat{\imath} \\
\tilde{0}\end{array}$ & $\overrightarrow{\widehat{\sigma}}$ & $\left|\begin{array}{c}0 \\
m \\
m\end{array}\right|$ & $\begin{array}{l}2 \\
i \\
i\end{array}$ & $\left|\begin{array}{l}\vec{b} \\
i\end{array}\right|$ & $\begin{array}{c}0 \\
\cdots \\
\text { vi }\end{array} \mid$ & $\begin{array}{c}\tilde{c} \\
\hat{i}\end{array}$ & $\left|\begin{array}{c}1 \\
\infty \\
i\end{array}\right|$ & $\left|\begin{array}{c}1 \\
0 \\
0\end{array}\right|$ & $\hat{n}$ & $\begin{array}{c}0 \\
n \\
i\end{array} \mid$ & ڤิ) & $\begin{array}{l}\text { के } \\
\text { nీ }\end{array}$ & 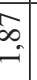 & \\
\hline & & ' & 1 & $\begin{array}{l}0 \\
m \\
0 \\
0\end{array}$ & ' & & $\begin{array}{l}\infty \\
0 \\
0\end{array}$ & $\frac{1}{0}$ & $\stackrel{2}{0}$ & ' & $\hat{\widehat{\gamma}}$ & $\frac{n}{0}$ & $\begin{array}{c}\bar{n} \\
\tilde{o}\end{array}$ & 1 & ' & $\begin{array}{l} \pm \\
0\end{array}$ & & $\begin{array}{l}\bar{\infty} \\
0 \\
0\end{array}$ & సે & $\begin{array}{l}\vec{\lambda} \\
0\end{array}$ & $\begin{array}{l}9 \\
\text { a } \\
0\end{array}$ & $\frac{\ddots}{0}$ & & ' & 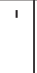 & ' & \\
\hline
\end{tabular}

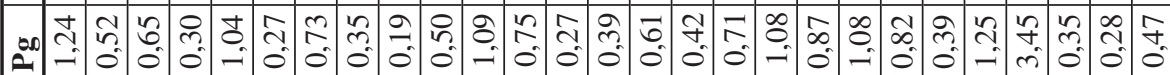

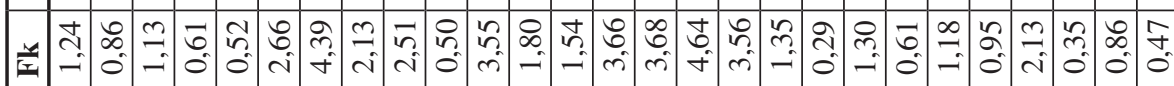
* กิ

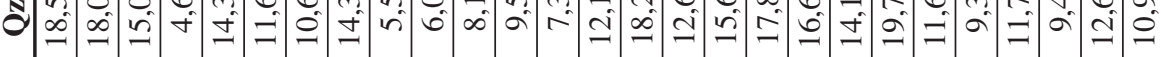

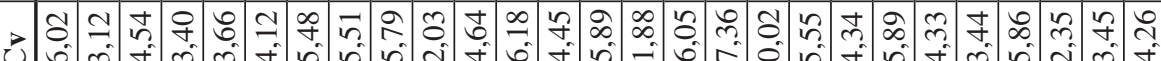

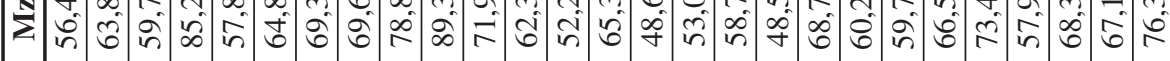


Relaciones de la Sociedad Argentina de Antropología 46 (2), julio-diciembre 2021: 503-525

\begin{tabular}{|c|c|c|c|c|c|c|c|c|c|c|c|c|c|c|c|c|c|c|c|c|c|c|c|c|c|c|c|c|}
\hline ڤิ & & $\begin{array}{l}\mathcal{N} \\
\mathfrak{O}^{\circ}\end{array}$ & $\begin{array}{l}0 \\
0 \\
0\end{array}$ & $\begin{array}{l}\infty \\
+ \\
+ \\
0 \\
0\end{array}$ & $\begin{array}{l}0 \\
\infty \\
0\end{array}$ & $\begin{array}{c}\infty \\
0\end{array}$ & \begin{tabular}{|l|}
$\infty$ \\
$0^{\circ}$
\end{tabular} & $\begin{array}{l}\dot{\sigma} \\
0 \\
0\end{array}$ & $\begin{array}{l}\tilde{O} \\
0 \\
0\end{array}$ & $\tilde{\sigma}$ & $\begin{array}{l}J_{0} \\
0 \\
0\end{array}$ & : & $\frac{n}{0}$ & $\begin{array}{l}F_{0} \\
\sigma_{0}\end{array}$ & $\begin{array}{l}\tilde{2} \\
\tilde{0} \\
0\end{array}$ & 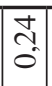 & 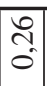 & $\begin{array}{ll} \\
0 \\
0\end{array}$ & $\begin{array}{l}2 \\
0 \\
0\end{array}$ & $\begin{array}{l}n \\
\tilde{n} \\
0\end{array}$ & $\begin{array}{c}\tilde{N} \\
0\end{array}$ & 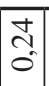 & $\begin{array}{l}n \\
\approx \\
-1\end{array}$ & \begin{tabular}{l|}
0 \\
0 \\
+ \\
0
\end{tabular} & 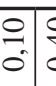 & $\begin{array}{l}e_{1} \\
\underbrace{}_{0}\end{array}$ & $\begin{array}{l} \pm \\
0^{\circ}\end{array}$ & $\frac{0}{c}$ \\
\hline & 1 & 1 & 1 & 1 & 1 & 1 & ' & & 1 & 1 & I & 1 & 1 & & 1 & & 1 & 1 & & 1 & $\stackrel{\imath}{2}$ & & & & & & & \\
\hline & ' & 1 & $\begin{array}{c}0 \\
0 \\
0\end{array}$ & 1 & & 1 & $\begin{array}{c}\infty \\
0 \\
0\end{array}$ & $\begin{array}{l} \pm \\
0 \\
0\end{array}$ & $\hat{n}$ & $\delta$ & $\mid \begin{array}{l}0 \\
0 \\
0\end{array}$ & $\mid \begin{array}{l}\vec{\lambda} \\
0\end{array}$ & & & & & & & $\frac{9}{0}$ & $\begin{array}{l}0 \\
0 \\
-1\end{array}$ & $\begin{array}{l}0 \\
+ \\
0\end{array}$ & & & 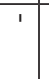 & & & $\frac{ \pm}{0}$ & \\
\hline & ' & $\frac{ \pm}{0}$ & $\begin{array}{c}1 \\
\tilde{2} \\
0\end{array}$ & $\begin{array}{l}0 \\
0\end{array}$ & 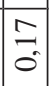 & $\stackrel{\infty}{*}$ & 1 & ' & ' & $\begin{array}{l}0 \\
+ \\
0 \\
0\end{array}$ & 8 & $\begin{array}{l}\vec{\jmath} \\
0\end{array}$ & 1 & $\begin{array}{l}\tilde{2} \\
0 \\
0\end{array}$ & $\begin{array}{l}\tilde{2} \\
0\end{array}$ & ' & 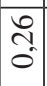 & ' & \begin{tabular}{|l|}
$\infty$ \\
$n$ \\
$n$ \\
0
\end{tabular} & $\begin{array}{l}2 \\
\infty \\
0\end{array}$ & $\begin{array}{l}\tilde{3} \\
\hat{0}\end{array}$ & ' & $\begin{array}{c}\hat{n} \\
0\end{array}$ & $\tilde{\sigma}$ & & & $\begin{array}{l} \pm \\
0^{\circ}\end{array}$ & (c) \\
\hline$y$ & 1 & 7 & 1 & 1 & 1 & 1 & I & 1 & 1 & ' & 1 & 1 & 1 & 1 & & 1 & 1 & 1 & T & $\begin{array}{l}n \\
0 \\
0\end{array}$ & & & 1 & 1 & & 1 & & \\
\hline 玨 & $\begin{array}{l}a \\
0 \\
0\end{array}$ & $\begin{array}{l}N \\
N \\
0\end{array}$ & $\begin{array}{l}0 \\
+ \\
+ \\
0\end{array}$ & $\mid \begin{array}{l}2 \\
2 \\
0 \\
0\end{array}$ & ' & $\begin{array}{c}0 \\
m \\
0 \\
0\end{array}$ & ' & m. & 1 & ' & & 1 & $\begin{array}{l}\sigma \\
0\end{array}$ & & 1 & \begin{tabular}{l}
+ \\
\multirow{0}{*}{}
\end{tabular} & 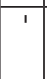 & 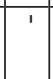 & \begin{tabular}{|c|}
$\hat{q}$ \\
$m$
\end{tabular} & $\begin{array}{l} \pm \\
+ \\
0\end{array}$ & . & & ' & $\begin{array}{l}\tilde{2} \\
0\end{array}$ & & 1 & & \\
\hline بـ & $\mid \begin{array}{l}- \\
\dot{J} \\
-\end{array}$ & $\left|\begin{array}{l}n \\
0 \\
n\end{array}\right|$ & $\begin{array}{l}8 \\
10\end{array}$ & $\begin{array}{l}0 \\
\infty \\
0 \\
0\end{array}$ & $\frac{n}{i}$ & $\begin{array}{l}8 \\
\infty \\
\infty\end{array}$ & $\begin{array}{l}\hat{n} \\
=\end{array}$ & $\begin{array}{l}尺 \\
+ \\
+\end{array}$ & $\begin{array}{l}\infty \\
0 \\
\text { i }\end{array}$ & $\hat{0}$ & $\mid \begin{array}{l}8 \\
0 \\
-1\end{array}$ & $\left|\begin{array}{l}\infty \\
\stackrel{1}{f} \\
\sim\end{array}\right|$ & o. & $\begin{array}{c}\hat{n} \\
i\end{array}$ & $\left|\begin{array}{l}\hat{\tilde{n}} \\
\hat{\imath}\end{array}\right|$ & $\left|\begin{array}{l}n \\
\hat{i} \\
\hat{i}\end{array}\right|$ & $\begin{array}{l}\hat{\alpha} \\
\infty\end{array}$ & $\left|\begin{array}{l}0 \\
\dot{d} \\
\dot{d}\end{array}\right|$ & $\begin{array}{l}\hat{N} \\
\dot{\sim} \\
\hat{n}\end{array}$ & $\begin{array}{l}0 \\
⿱ \\
r \\
r\end{array}$ & $\begin{array}{l}0 \\
0 \\
0\end{array}$ & ' & $\frac{\pi}{6}$ & $\begin{array}{l}\infty \\
\sigma^{\prime}\end{array}$ & 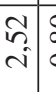 & $\begin{array}{l}\infty \\
\infty \\
0\end{array}$ & 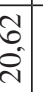 & n \\
\hline & $\frac{9}{0}$ & $\begin{array}{l}\mathscr{m} \\
\stackrel{0}{0}\end{array}$ & & $\begin{array}{l}0 \\
0\end{array}$ & $\begin{array}{l}\mathbf{T} \\
\text { s. } \\
0\end{array}$ & $\begin{array}{l}0 \\
2 \\
0 \\
0\end{array}$ & $\frac{\infty}{0}$ & $\begin{array}{c}\bar{m} \\
0\end{array}$ & $\begin{array}{l}\hat{\jmath} \\
\hat{0}\end{array}$ & $\begin{array}{l}n \\
\tilde{0}\end{array}$ & $\mid \begin{array}{c}1 \\
\hat{2} \\
0\end{array}$ & 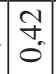 & ' & $\begin{array}{l}2 \\
\hat{0} \\
0\end{array}$ & $\begin{array}{l}\hat{2} \\
0\end{array}$ & 1 & $\mid \begin{array}{l}0 \\
1 \\
0 \\
0\end{array}$ & $\mid \begin{array}{l}n \\
0 \\
0\end{array}$ & $\mid \begin{array}{l}2 \\
0\end{array}$ & $\begin{array}{c}n \\
n \\
0 \\
0\end{array}$ & $\begin{array}{l}\tilde{2} \\
\hat{0}\end{array}$ & 1 & $\left|\begin{array}{l}\infty \\
\infty \\
0\end{array}\right|$ & $\begin{array}{l}\tilde{y} \\
\hat{0}\end{array}$ & $\begin{array}{ll}0 & 1 \\
0 & \end{array}$ & $\begin{array}{l}0 \\
0 \\
0\end{array}$ & $\begin{array}{l}\infty \\
\text { הె } \\
0\end{array}$ & \\
\hline & I & 1 & & $\frac{0}{0}$ & & 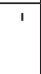 & ' & ' & & & & & & & & & 1 & & & & & & & & & & & \\
\hline & 1 & 1 & 1 & 1 & I & 1 & I & ' & ' & 1 & I & I & 1 & ' & 1 & ' & 1 & 1 & I & ' & 1 & 1 & I & ' & & ' & & \\
\hline$=$ & 1 & 1 & $\frac{0}{0}$ & 1 & 1 & 1 & ' & 1 & 1 & 1 & 1 & ' & 1 & $\tilde{\lambda}$ & & & ' & 1 & $\begin{array}{l}2 \\
0\end{array}$ & ' & $\tilde{n}$ & ' & 1 & 1 & & 1 & & \\
\hline & $\frac{a}{0}$ & 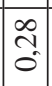 & $\begin{array}{l}0 \\
0 \\
0\end{array}$ & $\begin{array}{l}0 \\
0 \\
0\end{array}$ & $\frac{1}{0}$ & $\begin{array}{l}\infty \\
0 \\
0\end{array}$ & 1 & ' & ' & ' & $\because$ & 1 & $\begin{array}{l}0 \\
0 \\
0\end{array}$ & ' & 1 & 1 & $\begin{array}{l}\text { Ni } \\
\text { o. }\end{array}$ & ' & 1 & 1 & ' & I & ' & ' & & & $\begin{array}{l}\infty \\
\text { ปn } \\
0\end{array}$ & \\
\hline$\sum$ & $\left|\begin{array}{l}0 \\
\infty \\
m\end{array}\right|$ & 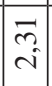 & $\begin{array}{l}0 \\
i \\
i\end{array}$ & $\stackrel{\mathrm{\imath}}{-}$ & $\bar{n}$ & $=$ & 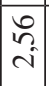 & $\stackrel{2}{\sim}$ & $\stackrel{2}{\sim}$ & ֶָ. & $\stackrel{\overbrace{}}{-}$ & $\begin{array}{l}n \\
\infty \\
0 \\
0\end{array}$ & થn & $\begin{array}{l}\hat{f} \\
0\end{array}$ & $\begin{array}{l}\stackrel{P}{m} \\
\text { m. }\end{array}$ & $\stackrel{-}{\approx}$ & $\frac{n}{\sim}$ & $\begin{array}{l}m \\
\tilde{i}\end{array}$ & $\mid \begin{array}{l}\infty \\
n \\
0 \\
0\end{array}$ & $\stackrel{0}{0}$ & $\begin{array}{l}0 \\
= \\
=\end{array}$ & 文 & 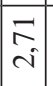 & $\begin{array}{c}\triangleright \\
\infty \\
i\end{array}$ & $\begin{array}{l}c \\
0 \\
i\end{array}$ & 8 & $\begin{array}{c}0 \\
m \\
m\end{array}$ & જ́ \\
\hline a & $\left|\begin{array}{l}\forall \\
\dot{8}\end{array}\right|$ & $\begin{array}{c}1 \\
0 \\
i\end{array}$ & $\begin{array}{l}8 \\
n \\
n\end{array}$ & $\begin{array}{l}\curvearrowright \\
\text { in }\end{array}$ & 0 & $\begin{array}{l}0 \\
n \\
i\end{array}$ & $\frac{\infty}{0}$ & $\stackrel{2}{\vec{i}}$ & $\hat{\jmath}$ & o. & $\begin{array}{c}0 \\
\dot{m}\end{array} \mid$ & $\underset{q}{\stackrel{g}{q}}$ & $\begin{array}{l}\text { o } \\
\text { ஸे }\end{array}$ & $\stackrel{\sim}{\mathcal{I}_{-}}$ & $\begin{array}{l}0 \\
0 \\
+ \\
0 \\
0\end{array}$ & $\begin{array}{l}\text { กิ } \\
\text { m. }\end{array}$ & $\begin{array}{l}\infty \\
\\
n\end{array}$ & a & S & $\underset{0}{0}$ & 足 & 交 & $\begin{array}{l}\vec{N} \\
\text { i }\end{array}$ & $\begin{array}{l}\mathcal{q}_{-} \\
-\end{array}$ & $\begin{array}{c}m \\
\tilde{c} \\
m\end{array}$ & $\nabla_{0}$ & $\begin{array}{l}\stackrel{y}{+} \\
0 \\
0\end{array}$ & $\vec{b}$ \\
\hline & & & & $\begin{array}{l}\mathbb{J} \\
0 \\
0\end{array}$ & & $\stackrel{\infty}{=}$ & $\frac{\infty}{0}$ & ' & & & $\approx$ & & & & & & & $\bar{m}$ & $\stackrel{2}{2}$ & & $\subseteq$ & & & $\begin{array}{l}\tilde{N} \\
\tilde{0}\end{array}$ & & & $\frac{ \pm}{0}$ & 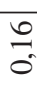 \\
\hline
\end{tabular}

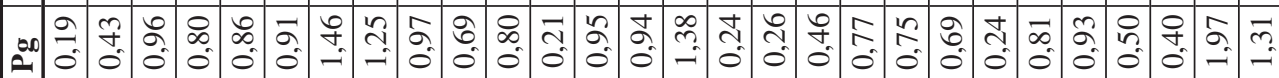

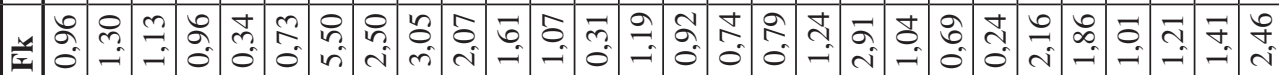

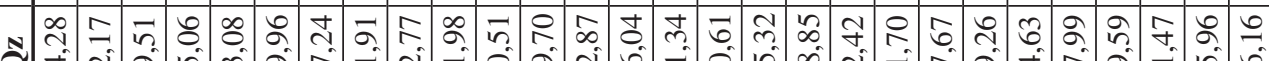

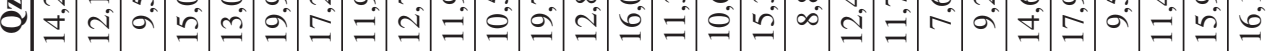

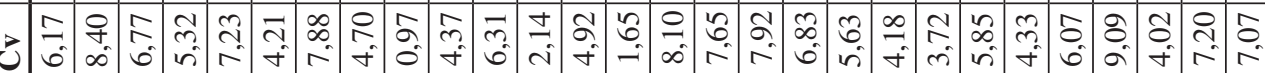
ง

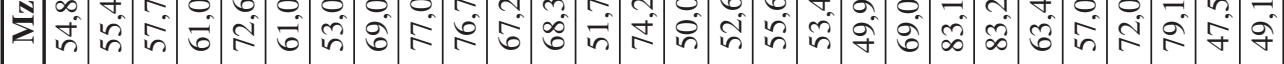

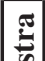
ڤ్⿹ $\stackrel{8}{\frac{8}{3}}$

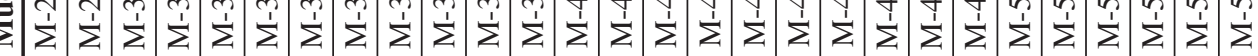


El Grupo 3 (M-8, 23, 25, 26, 29, 30, 31, 32, 35, 47 [tablas 1 y 2 y figuras 6 y 9]) está configurado por pastas gruesas y medias que presentan como material no plástico: cuarzo (9\%-15\%), feldespato potásico $(\leq 2,5 \%)$, plagioclasas $(\leq 1,25 \%)$, biotita $(0,7 \%-6 \%)$, muscovita $(0,5 \%-2,3 \%)$, anfíboles-piroxenos $(\leq 0,6 \%)$, litoclastos graníticos $(0,9 \%-15,7 \%)$, esquisto $(\leq 1,6 \%)$, litoclastos volcánico $(\leq 0,9 \%)$, trizas volcánicas $(\leq 0,9 \%)$, minerales opacos $(\leq 1,5 \%)$. Las inclusiones oscilan entre $20 \%$ y $36 \%$ del total de la pasta y principalmente corresponden a agregado de una arena seleccionada de granulometría gruesa, entre $500 \mu \mathrm{m}$ y $2000 \mu \mathrm{m}$ (arena gruesa-arena muy gruesa). Las texturas de estas pastas se destacan por presentar estructura de fondo microgranosa, algo pseudolepidoblástica. La matriz representa entre 55\% y $73 \%$ del total de la pasta. Las inclusiones tienen formas subangulares y subredondeadas. Las cavidades son escasas, constituyendo entre 2,3\% y $8,4 \%$. Esto podría estar señalando el carácter resistente de estos recipientes. La coloración castaña clara del fondo de pasta estaría indicando que las piezas fueron cocidas en ambiente oxidante.

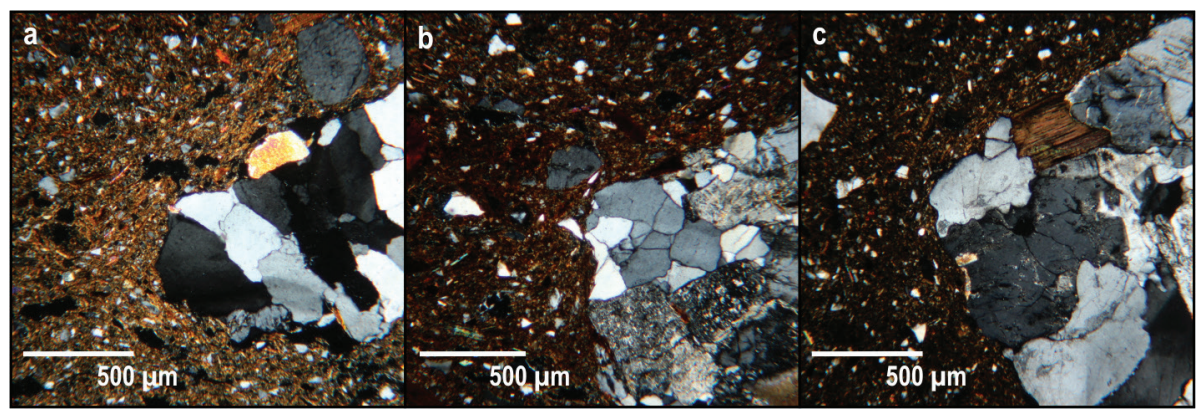

Figura 9. Fotomicrografías de pastas del Grupo 3: a) M-8; b) M-29; c) M-47

El Grupo 4 (M-4, 7, 9, 11, 38, 51 [tabla 1 y 2 y figuras 6 y 10]) está compuesto por pastas medias que presentan como material no plástico: cuarzo (4,6\%-18\%), feldespato potásico $(0,6 \%$ $4,4 \%)$, plagioclasas $(\leq 1,1 \%)$, biotita $(0,3 \%-3 \%)$, muscovita $(0,3 \%-2,8 \%)$, anfíboles-piroxenos $(\leq 0,5 \%)$, litoclastos graníticos $(2,4 \%-9,8 \%)$, esquisto $(\leq 0,2 \%)$, litoclastos volcánico $(0,9-2,5 \%)$, trizas volcánicas $(\leq 0,9 \%)$, minerales opacos $(\leq 0,6 \%)$. Las inclusiones oscilan entre $11 \%$ y $37 \%$ del total de la pasta y principalmente corresponden a agregado de un sedimento seleccionado de granulometría media, entre $250 \mu \mathrm{m}$ y $1000 \mu \mathrm{m}$ (arena media-arena gruesa). Las texturas de estas pastas se destacan por presentar estructura de fondo criptofilitosa, algo pseudolepidoblástica. La matriz representa entre $57 \%$ y $85 \%$ del total de la pasta. Las inclusiones tienen formas subangulares y subredondeadas. Las cavidades son escasas, constituyendo entre 3,4\% y 6,3\%. Esto podría estar señalando el carácter resistente de estos recipientes. La coloración castaña rojizo oscuro del fondo de pasta estaría indicando que las piezas fueron cocidas en ambiente oxidante.

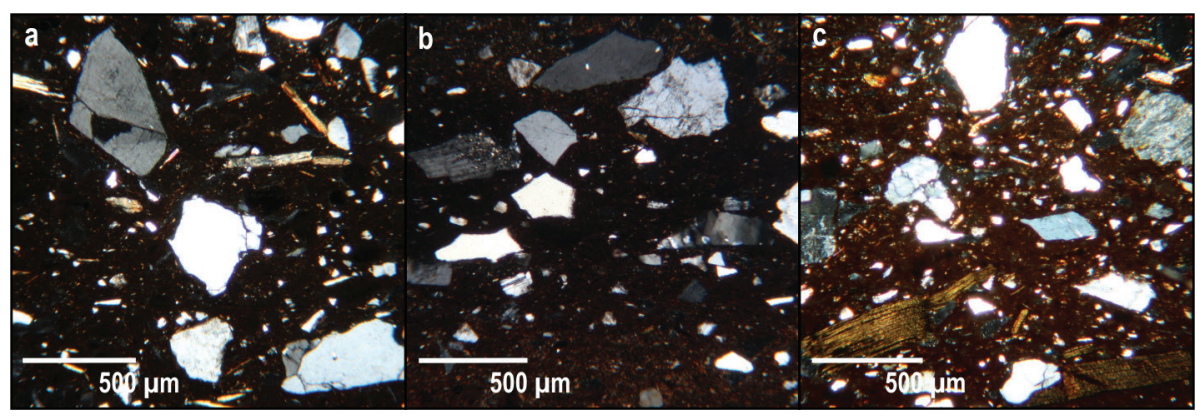

Figura 10. Fotomicrografías de pastas del Grupo 4: a) M-9; b) M-11; M-38 
El Grupo 5 (M-10, 19, 27, 36, 37, 39, 41, 48, 49, 52, 53 [tablas 1 y 2 y figuras 5, 6 y 11]) está conformado por pastas finas que presentan como material no plástico: cuarzo $(6 \%-20 \%)$, feldespato potásico $(0,2 \%-3 \%)$, plagioclasas $(\leq 1 \%)$, microclino $(\leq 0,5 \%)$, biotita $(0,5 \%-3 \%)$, muscovita $(0,2 \%-2 \%)$, anfíboles-piroxenos $(\leq 0,4 \%)$, esquisto $(\leq 0,3 \%)$, litoclastos graníticos $(\leq$ $4,2 \%)$, litoclastos volcánicos $(\leq 0,5 \%)$, trizas volcánicas $(\leq 0,9 \%)$, minerales opacos $(\leq 1 \%)$. Las inclusiones oscilan entre $8 \%$ y $29 \%$ del total de la pasta y principalmente corresponden a agregado de una arena seleccionada de granulometría fina, entre $30 \mu \mathrm{m}-250 \mu \mathrm{m}$ (limo medio-arena fina). Aunque también se observaron algunos clastos de mayor tamaño. Las texturas de estas pastas se destacan por presentar estructura de fondo pseudolepidoblástica algo criptofilitosa. La matriz representa entre $68 \%$ y $89 \%$ del total de la pasta. Las inclusiones tienen formas subangulares y subredondeadas. Las cavidades son escasas, constituyendo entre 1\% y $9 \%$. Esto podría estar señalando el carácter resistente de estos recipientes.

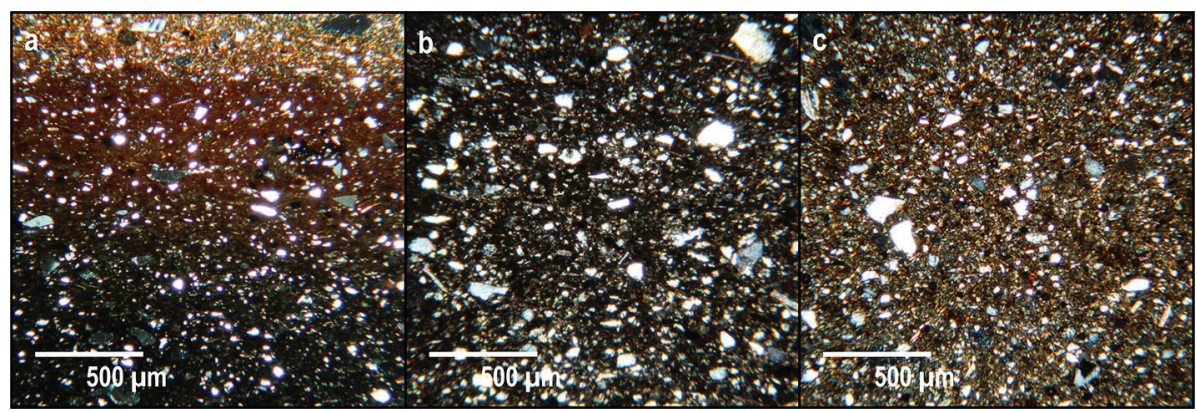

Figura 11. Fotomicrografías de pastas del Grupo 5: a) M-10; b) M-36; c) M-39

El Grupo 6 está conformado hasta el momento por dos pastas gruesas (M-24, 40 [tablas 1 y 2 y figuras 5 y 12]) que se destacan por presentar una abundancia relativa de biotita $(1,6 \%-5,4 \%)$ y muscovita (6,3\%-12,5\%). Se registraron además de estas inclusiones, cuarzo (11\%-13\%), feldespato potásico $(0,3 \%-2 \%)$, plagioclasas $(1 \%-3,5 \%)$, litoclastos graníticos $(4,8 \%-9,7 \%)$ y minerales opacos $(\leq 0,2 \%)$. Además, la muestra 40 tiene $7 \%$ de esquisto. Las inclusiones oscilan entre $36 \%$ y $44 \%$ del total de la pasta. En la muestra 40, los elementos no plásticos se corresponden a un agregado de una arena no seleccionada cuya granulometría oscila entre $30 \mu \mathrm{m}-4000 \mu \mathrm{m}$ (limo medio-canto pequeño). En cambio, la muestra 24 tiene una granulometría predominante que oscila entre 250 $\mu \mathrm{m}$ y $1000 \mu \mathrm{m}$ (arena media-arena gruesa), es decir, posiblemente la ollera u ollero haya usado una arena seleccionada como temperante. Las texturas de estas pastas se destacan por presentar estructura de fondo pseudolepidoblástica. La matriz representa entre 52\% y 58\% del total de la pasta. Las inclusiones tienen formas subangulares y subredondeadas. Las cavidades son escasas, constituyendo entre $5 \%$ y $6 \%$. Esto estaría señalando el carácter resistente de estos recipientes.

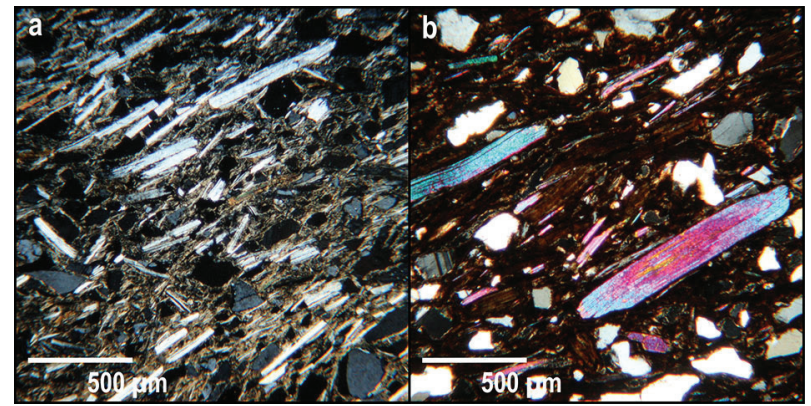

Figura 12. Fotomicrografías de pastas del Grupo 6: a) M-24; b) M-40 


\section{DISCUSIÓN}

El Grupo 1 reúne los fragmentos que tienen pastas gruesas con abundantes inclusiones con las que se elaboraron recipientes asignables a distintos tipos cerámicos como Gris Pulido Inciso, Ante Alisado Inciso, Blanco sobre Rojo e Inciso, Ordinario Alisado, Ordinario Alisado Inciso y Pintado, Ordinario Alisado de Pared Delgada, Ordinario Alisado Inciso de Pared Delgada, Ordinario Pintado, Alumbrera Líneas Paralelas, Alumbrera Post-cocción, Alumbrera Tricolor. Comparativamente, tiene semejanzas con las pastas gruesas de la tradición Tafí (Cremonte 1996) y la tradición alfarera del sur de los valles Calchaquíes (Pereyra Domingorena 2012, 2015).

El Grupo 2 se caracteriza por aunar las pastas con densidad moderada, en las que se registraron inclusiones de un amplio rango granulométrico, es decir que las alfareras y alfareros habrían utilizado arenas no seleccionadas para incorporar a las arcillas. Estas pastas fueron observadas en fragmentos de los tipos Condorhuasi, Gris Pulido Inciso, Rojo Sobre Ante, Alumbrera Pintado y Ordinario Alisado. Este patrón de pasta también fue registrado en los yacimientos arqueológicos del Período Formativo del faldeo occidental del Aconquija, el valle del Cajón y el valle de Santa María (Pereyra Domingorena 2012, 2015; Pereyra Domingorena y Bugliani 2019).

El Grupo 3 está constituido por pastas con elementos no plásticos grandes, pero poco abundantes, es decir que se habría utilizado una arena gruesa seleccionada para las manufacturas de estos recipientes, los cuales fueron clasificados como Condorhuasi, Ordinario Alisado y Ordinario Pintado. Un modo de pasta similar se ha detectado también en la tradición alfarera del sur de los valles Calchaquíes (Pereyra Domingorena 2012, 2015).

La presencia de inclusiones de esquisto (en baja proporción relativa), entre los casos de los grupos 1, 2 y 3, podría estar indicando que estas vasijas fueron manufacturadas de manera local en el área de Campo del Pucará, correspondiendo quizá a materiales líticos del sustrato geológico del área (González Bonorino 1950). Esta hipótesis de trabajo deberá se contrastada con estudios de arenas locales y conformación de series cerámicas experimentales.

En el Grupo 4 se reconocieron fragmentos Gris Pulido, Ciénaga Negro sobre Crema, Ciénaga Negro sobre Ante, Baño Blanco Pulido Inciso, Ordinario Alisado Pared Delgada. Este patrón de pastas es semejante a los modos de pastas con inclusiones de granulometría arena media registrados en Tafí (Cremonte 1996) y el sur de los valles Calchaquíes (Pereyra Domingorena 2012, 2015; Pereyra Domingorena y Bugliani 2019).

El Grupo 5 reúne pastas finas en las que se usó temperante de arena muy fina para realizar el barro para manufacturar los recipientes. Se elaboraron con este tipo de pasta vasijas asignadas a los tipos Ciénaga Negro sobre Ante, Gris Pulido Inciso, Gris Pulido Grabado, Negro Pulido Inciso, Condorhuasi Polícromo y Ordinario Pintado. Estas pastas finas están emparentadas con otras producciones del Noroeste argentino como las catalogadas en el sur de los valles Calchaquíes (Pereyra Domingorena 2012, 2015; Pereyra Domingorena y Bugliani 2019) y el valle de Tafí (Cremonte 1996). El aumento de muestras cerámicas analizadas petrográficamente de sitios Alamito podría reconfigurar este agrupamiento de las pastas finas.

El grupo 6 está asociado a fragmentos del tipo Gris Pulido Inciso (M-24, figura 2.e) y del tipo Ordinario Alisado. Las pastas con abundancia de micas registradas por petrografía, hasta el momento, están vinculadas mayormente con "los estilos Inca Provincial, principalmente Inca Rojo y Blanco, Inca Indeterminada, ordinaria y ollas con pie de compotera Caspinchango" (Marchegiani 2012:86); con un fragmento Santa María Negro sobre Crema (Cremonte y Pereyra Domingorena 2013), hallado en el sitio de Tambo de Angastaco; con cerámica de estilos Santa María (Castellanos et al. 2019); y con el conjunto Micáceo detectado en Peña del Medio (Puente 2020). Esto parecería obedecer a un fenómeno técnico del Período Tardío y de la dominación incaica. Sin embargo, algunas muestras correspondientes al grupo petrográfico 8 de Antofagasta de la Sierra (Gasparoti y Escola 2020) y los casos aquí analizados podrían sugerir que este tipo 
particular de pasta ya se estaba usando en el Período Formativo. En tal caso, habría que examinar el tipo cerámico Alumbrera Ordinario descripto para los sitios Alamito que presentaría -según las observaciones macroscópicas- abundante micas en sus pastas (Núñez Regueiro 1998). Estudios posteriores, que involucren mayor cantidad de muestras de tipos cerámicos de los períodos Formativo, Tardío y de momentos de la ocupación incaica y de distintas áreas del Noroeste argentino, podrán profundizar el conocimiento sobre esta particular forma de elaborar los barros alfareros prehispánicos.

Las pastas cerámicas de los sitios Alamito, aquí estudiadas, se caracterizan por tener una mayor abundancia relativa -en comparación con otros tipos de pastas del Período Formativo del Noroeste argentino- de material no plástico de granulometría limo grueso-arena muy fina (30 $\mu \mathrm{m}-125 \mu \mathrm{m})$. Esto hace suponer que, quizá, las antiguas alfareras y alfareros usaron arcillas con un contenido natural de material no plástico fino relativamente mayor al registrado en áreas vecinas (Pereyra Domingorena 2012, 2015). Esta idea también se refuerza porque se observan en los nódulos de arcillas -reconocidos en algunos de los cortes- inclusiones muy pequeñas. Los agrupamientos tentativos, cuyos atemperantes mayoritarios están representados por cristaloclastos de cuarzo y litoclastos graníticos -a excepción del Grupo 6- y que presentan, en algunos ejemplares, una baja proporción relativa de inclusiones de esquisto podrían corresponder entonces a una tradición de manufactura del área del Campo del Pucará. Se habría conformado así una manera particular de hacer objetos que implicó saber sobre las fuentes de abastecimiento de arenas, arcillas y leñas; conocer estas cinco recetas para la preparación de los barros; la destreza para el levantado de piezas; la experticia con pulidores, herramientas para hacer incisiones y grabados, pinceles; etc., es decir, el proceso productivo, las habilidades y el conocimiento práctico (Mauss 1979 [1935]; Lemonnier 2002 [1993]; Stark 1999) que tuvieron las alfareras y alfareros de los sitios Alamito durante los primeros siglos d.C. La singularidad del Grupo 6 por ahora no permite postular un lugar de producción particular en el Noroeste argentino, aunque no se descarta como hipótesis una producción local en las aldeas Alamito.

Los agrupamientos de pastas presentan similitudes con tradiciones alfareras detectadas en el valle del Tafí (Cremonte 1996) y el sur de los valles Calchaquíes (Pereyra Domingorena 2012, 2015), áreas próximas al Campo del Pucará (figura 1). En los valles de Tafí, Santa María y Cajón y en la Falda occidental del Aconquija se han descrito pastas gruesas con abundantes atemperantes de un amplio rango granulométrico; distintas clases de pastas medias, variando según los tamaños y proporciones de los elementos no plásticos; y de pastas finas, que se distinguen principalmente por el posible agregado inclusiones de tamaños finos y de pastas sin atemperantes. Las similitudes técnicas -pasta, morfología, diseños plásticos- y la cercanía permiten pensar en rasgos culturales compartidos en la producción alfarera que, a su vez, podría haber implicado una cosmovisión similar entre estas poblaciones durante los primeros siglos d.C. A su vez, la repetición a través del tiempo de estas disposiciones o modos de preparar los barros alfareros habría permitido, por un lado, la trasmisión específica de los conocimientos de las y los ceramistas en el quehacer propio de esta manufactura, pero también como un indicador material para la comunicación de otros saberes culturales generales de estas sociedades orales. En principio, la cerámica vincula a los habitantes de los sitios Alamito con otras comunidades aldeanas del Período Formativo del actual Noroeste argentino, aunque las formas constructivas, la morfología, la distribución espacial y el patrón de abandono de las casas en Campo del Pucará (Gordillo y Leiton 2015) remitirían a prácticas culturales propias del área.

Además, estas similitudes se pueden observar en la alfarería del estilo Condorhuasi. En investigaciones previas se examinaron muestras elaboradas con pastas gruesas de densidad moderada y pastas medias de arenas gruesas seleccionadas (Pereyra Domingorena 2012, 2015), que también se describieron en fragmentos Condorhuasi hallados en los sitios Alamito. Lo novedoso 
resultó haber encontrado tiestos de este estilo, manufacturados con una pasta fina. Esta cualidad técnica no había sido aún registrada de manera petrográfica, pero sí mencionada en los trabajos de Antonio Serrano (1944) y Alberto Rex González (1956).

También, se pudieron observar ciertas semejanzas a nivel de las proporciones de los atemperantes con producciones de otras áreas vecinas, aunque las diferencias petrográficas plantearían que las piezas aquí analizadas no circularon de estas regiones. Por ejemplo, las producciones cerámicas de Período Formativo del valle de Hualfín presentarían una relativa abundancia de inclusiones de vidrio vesicular y litoclastos volcánicos (Zagorodny y Balesta 2005), hecho que no se ha reconocido en los materiales estudiados de Alamito. Esto permite pensar que los casos estilísticamente adscriptos a los tipos cerámicos Ciénaga -incisos y pintados-serían manufacturas locales en el Campo del Pucará. Futuros estudios, que por ejemplo focalizaran en estos tipos cerámicos particulares, podrían detectar materiales elaborados en el valle de Hualfín. También pudimos correlacionar esta investigación con producciones de la Puna catamarqueña a través de los datos obtenidos en la zona de Laguna Blanca y en la región de Antofagasta de la Sierra. En la primera de ellas, la información que se cuenta expone la presencia de rocas efusivas con textura esferulítica entre las inclusiones de las pastas cerámicas (Espiro 2013). Este tipo específico de litoclasto no ha sido reportado como temperante en las muestras examinadas de Alamito. En cuanto al área de Antofagasta de la Sierra, los estudios petrográficos sobre la alfarería del Formativo indican que hay una gran variabilidad de atemperantes usados en las pastas cerámicas, entre los que se destacan los cristales de cuarzo en combinación con distintas rocas de origen volcánico y/o plutónico, plagioclasas con y sin alteraciones a seriecita, muscovita y feldespatos alterados (Gasparotti y Escola 2020). Las combinaciones de material no plástico registradas en el área de Antofagasta de la Sierra tampoco han sido halladas en los sitios Alamito. Entonces, el conocimiento actual no permite afirmar que hubiera circulación de piezas alfareras desde Laguna Blanca y Antofagasta de la Sierra y hacia el Campo de Pucará.

Asimismo, los resultados de los análisis petrográficos aquí presentados demuestran que no hay similitudes técnicas a nivel de las pastas con las producciones alfareras de la cuenca Rosario-Lerma (Quebrada del Toro y valle de Lerma) y del valle del río San Francisco. En el primer caso, la cerámica que ha sido asociada a producción local presenta litoclastos metamórficos de bajo grado (pizarra y filita), tiesto molido y arenisca (De Feo y Pereyra Domingorena 2018) y no ha sido descripto este tipo de inclusiones en las muestras aquí estudiadas. En el segundo caso, no se ha podido correlacionar ningún tiesto con los cuatro grupos de pastas asociados a la producción de la Tradición San Francisco (Cremonte et. al. 2016), que también se caracteriza por el uso de tiesto molido para la confección de los barros alfareros. Es decir, las alfareras y alfareros de Campo del Pucará no habrían tenido la intencionalidad de emplear tiesto molido como atemperante y estarían más vinculados, es este sentido, con las tradiciones alfareras valliserranas del Período Formativo del Noroeste argentino. Además, se puede pensar -con la evidencia presentada hasta el momento- que los habitantes de las aldeas de Alamito no participaron de los circuitos de intercambio con estas dos regiones del actual Noroeste argentino o, mejor dicho, si estas sociedades estaban insertas en estas redes, entonces, debieron circularon bienes distintos a los objetos hechos con arcilla.

Finalmente, este estudio dio como resultado una clasificación que servirá como base para futuras investigaciones que procuren profundizar los análisis petrográficos de las pastas cerámicas, los estilos técnicos involucrados en la confección y otras aristas de la producción alfarera de las poblaciones prehispánicas que habitaron en los sitios Alamito del Campo del Pucará y sus vinculaciones con otras sociedades del Período Formativo del Noroeste argentino. 


\section{CONCLUSIONES}

La investigación permitió establecer que la cerámica consumida en los sitios Alamito fue manufacturada con seis modos técnicos. Cinco de los cuales presentan semejanzas con las producciones alfareras del valle de Tafí y del sur de los valles Calchaquíes desarrolladas en los primeros siglos d.C. y con particularidades locales. La evidencia hasta aquí presentada no permite pensar en la producción alóctona de los materiales analizados y su circulación al área de Campo del Pucará. Hasta el momento no se ha detectado tiesto molido en las muestras analizadas, esto diferencia la producción, el consumo y la circulación de la alfarería de Alamito de otros sectores de la región valliserana del Noroeste argentino durante el Período Formativo, donde sí se han observado manufacturas locales o cerámicas alóctonas producidas en con este atemperante. Asimismo, hay que continuar con los estudios para llegar a determinar si hay correlación entre las morfologías de las piezas del estilo Condorhuasi y la diversidad de pastas registradas en esta particular cerámica de los primeros siglos d.C. del Noroeste argentino.

El estudio también buscó entender las antiguas técnicas alfareras -entendidas como las relaciones entre las materias primas, las habilidades y las destrezas- que sirven para la reproducción social, a través de transmisión social del aprendizaje cerámico y la memoria colectiva. Se propone a futuro la necesidad de ahondar y contrastar las hipótesis aquí enunciadas con un número mayor de muestras cerámicas. Además, es preciso investigar la correlación entre las formas de las vasijas, los otros tipos cerámicos del área, los grupos de pastas aquí propuestos y sus posibles futuras modificaciones. Quizá, nuevos estudios ayudarán a comprender la circulación de bienes cerámicos que no ha podido ser revelada a partir de las muestras analizadas.

Finalmente, este trabajo puso en valor una antigua colección arqueológica, revalorizando el acervo del Museo de Antropología de Córdoba, a través de conceptualizaciones teóricas novedosas y técnicas analíticas no habituales durante las investigaciones realizadas por el Dr. Núñez Regueiro.

\section{AGRADECIMIENTOS}

Al Museo de Antropología de la Universidad de Córdoba (Argentina) por autorizar la selección de las muestras cerámicas para los análisis petrográficos. Este trabajo de investigación se financió a través de los subsidios: PICT 2011 Raíces 633 (Lic. Scattolin); AHRC Early Career Grant SX-05317 (2011-2013), dirigido por la Dra. Lazzari; y PICT 2011 Jóvenes Investigadores 260 (Dr. Pereyra Domingorena).

Mi agradecimiento a las evaluadoras o evaluadores por la lectura crítica del manuscrito y los comentarios sugeridos, que han permitido una mejora sustancial de la versión original.

\section{NOTAS}

1 Para el análisis petrográfico de las inclusiones no plásticas se usaron los siguientes libros de referencia Yardley (1989), Best (2003), Klein y Hurlbut Jr (1998) y Kerr (1965).

\section{REFERENCIAS BIBLIOGRÁFICAS}

Adams, A. E., W. S. Mackenzie y C. Guilford (1997) [1984]. Atlas de rocas sedimentarias. Barcelona, Masson.

Angiorama, C. I. (1996-98). Nuevos aportes a la cronología de Condorhuasi-Alamito. Palimpsesto. Revista de Arqueología 5: 100-105. 
LuCAs Pereyra Domingorena - Aproximación a la ProducCión alfarera en los Sitios alamito duRANTE ...

Balfet, H, M. F. Fauvet-Berthelot y S. Monzon (1983). Pour la normalisation de la description des poteries. Paris, Editions du Centre National de la Recherche Scientifique.

Best, M. G. (2003). Igneous and Metamorphic Petrology. Oxford, Blackwell Publishing.

Bourdieu, P. (1988) [1979]. La distinción. Criterio y bases sociales del gusto. Madrid, Editorial Taurus.

Bourdieu, P. (1999) [1997]. Meditaciones pascalianas. Barcelona, Anagrama.

Bourdieu, P. (2007) [1980]. El sentido práctico. Buenos Aires, Siglo XXI.

Bugliani, M. F. (2008). Consumo y representación en el Formativo del sur de los Valles Calchaquíes (Noroeste argentino): Los conjuntos cerámicos de las aldeas del primer milenio A.D. Oxford, John and Erica Hedges Ltd.

Castellanos, M. C., M. F. Quiroga y A. A. Nieves (2019). Alfarería del prehispánico Tardío en las quebradas altas del Calchaquí (Salta): una primera aproximación a los estudios de pastas de Tacuil y Gualfín. Revista del Museo de Antropología 12 (3): 7-22.

Cremonte, M. B. (1996). Investigaciones arqueológicas en la quebrada de La Ciénega. (Dpto. de Tafí, Tucumán). Tesis Doctoral inédita; La Plata: Facultad de Ciencias Naturales y Museo, Universidad Nacional de La Plata.

Cremonte, M. B. y L. Pereyra Domingorena (2013). Atlas de pastas cerámicas arqueológicas. Petrografía de estilos alfareros del NOA. San Salvador de Jujuy, EdiUNJu.

Cremonte, M. B., L. Pereyra Domingorena y A. Scaro (2016). San Francisco, pastas cerámicas de una tradición alfarera de las yungas jujeñas. En S. Alconini (ed.), Entre la vertiente tropical y los valles. Sociedades regionales e interacción prehispánica en los Andes Centro-Sur: 241-261. La Paz, Ediciones Plural.

Courtois, L. (1976). Examen au microscope pétrographique des céramiques archéologiques. Notes et monographies techniques No. 8. Paris, Editions du Centre National de la Recherche Scientifique.

Culbert, P. y R. L. Rands (2007). Multiple classifications: an alternative approach to the investigation of Maya ceramics. Latin American Antiquity 18 (2): 181-190.

De Feo, M. E. y L. Pereyra Domingorena (2018). Interacciones sociales durante el Formativo: una mirada desde el análisis de la alfarería de la Quebrada del Toro. Arqueología Iberoamericana 40: 65-80.

Espiro, V. E. (2013). Comparando alfarerías de contextos domésticos de la aldea Piedra Negra para mediados y finales del primer milenio d.C. (Laguna Blanca - Catamarca). Arqueología 19 (1): 87-105.

Gasparotti, L. y P. Escola (2020). Tejiendo relaciones a través de la cerámica en los primeros momentos de la era en la puna meridional argentina. Estudios Atacameños 64: 243-275.

Gordillo, I. y D. Leiton (2015). El abandono en las sociedades formativas del Noroeste argentino. Casos y discusión. En M. A. Korstanje, M. Lazzari, M. Basile, M. F. Bugliani, V. Lema, L. Pereyra Domingorena y M. Quesada (eds.), Crónicas materiales precolombinas Arqueología de los primeros poblados del Noroeste Argentino: 635-661. Buenos Aires, Sociedad Argentina de Antropología.

Gosselain, O. P. (1992). Technology and Style: Potters and Pottery among Bafia of Cameroon. Man 27: 559-586.

Gosselain, O. P. (1999). In pots we trust. The processing of Clay and Symbols in Sub-Saharan Africa. Journal of Material Culture 4: 205-230. 
Relaciones de la Sociedad Argentina de Antropología 46 (2), julio-diciembre 2021: 503-525

González, A. R. (1956). La cultura Condorhuasi del Noroeste Argentino (apuntes preliminares para su estudio). Runa VII: 37-85.

González Bonorino, F. (1950). Descripción geológica de la Hoja 13e, Villa Alberdi (Boletín Nº 74). Buenos Aires, Dirección Nacional de Minería.

Hammer, Ø., D. A. T. Harper y P. D. Ryan (2001). PAST: Palaeontological statistics software package for education and data analysis. Palaeontologia Electronica 4 (1): 9.

Hosler, D. (1996). Technical choice, social categories and meaning among the Andean potters of Las Animas. Journal of Material Culture 1 (1): 63-92.

Kerr, P. F. (1965). Mineralogía óptica. Nueva York, McGraw-Hill Book Company.

Klein, C. y C. S Hurlbut Jr. (1998). Manual de mineralogía. Barcelona, Editorial Reverté.

Lemonnier, P. (2002) [1993]. Introduction. En P. Lemonnier (ed.), Technological choices. Transformation in material cultures since the Neolithic: 1-35. Londres, Routledge.

Marchegiani, M. (2012). La variabilidad alfarera en el tambo de Punta de Balasto y la producción cerámica en Yocavil en la época de la dominación incaica (Catamarca, Noroeste argentino). Arqueología 18: 77-100.

Mauss, M. (1979) [1935]. Técnicas y movimientos corporales. En Sociología y antropología: 337-356. Madrid, Editorial Tecnos.

Middleton, A., M. N. Leese y M. R. Cowell (1991). Computer assisted Approaches to the Grouping of Ceramic Fabrics. En A. Middleton y I. C. Freestone (eds.), Recent Development in Ceramic Petrology: 265276. Londres, British Museum Publications Ltd.

Miller, D. (1985). Artefacts as categories. Cambridge, Cambridge University Press.

Núñez Regueiro, V. A. (1998). Arqueología, historia y antropología de los sitios de Alamito. San Miguel de Tucumán, INTERDEA.

Ong, W. (2006) [1982]. Oralidad y escritura. Tecnologías de la palabra. México, Fondo de Cultura Económica.

Orton C., P. Tyers y A. Vince (1997). La cerámica en arqueología. Barcelona, Crítica.

Pereyra Domingorena, L. (2012). Manufacturas alfareras al sur de los valles Calchaquíes entre el siglo primero al quinto D.C. Relaciones 37 (2): 387-412.

Pereyra Domingorena, L. (2015). Estudio petrográfico de la cerámica arqueológica del primer milenio d.C. al sur de los valles Calchaquíes (Noroeste argentino). Chungara 47 (3): 415-428.

Pereyra Domingorena y M. F. Bugliani (2019). Las vasijas de Bordo Marcial: una caracterización integradora de un conjunto alfarero del Período Formativo en el Noroeste argentino. Intersecciones en Antropología 20 (2): 255-266.

Pérez Gollán, J. A. y M. Dujovne (1995). El Museo Etnográfico de la Facultad de Filosofía y Letras: balance de una gestión. Runa 22: 119-121.

Puente, V. (2012). Atravesando fronteras. Prácticas compartidas e identidades sociales negociadas durante el Tardío prehispánico. Una discusión desde la alfarería ordinaria del valle del Bolsón (Belén, Catamarca). Relaciones 37 (1): 65-87. 
Puente, V. (2020). La cerámica de la Peña del Medio, Paicuqui: variabilidad en un paisaje de encuentro (Antofagasta de la Sierra, Argentina). Relaciones 45 (2): 245-270.

Serrano, A. (1944). La cerámica tipo Condorhuasi y sus correlaciones. Publicaciones VI: 3-31.

Shennan, S. (1992) [1988]. Arqueología cuantitativa. Barcelona, Crítica.

Stark, M. T. (1999). Social dimensions of technical choice in Kalinga ceramic traditions. En E. S. Chilton (ed.), Material meanings. Critical approaches to the interpretation of material culture: 24-43. Salt Lake City, The University of Utah Press.

Tilley, C. (1999). Metaphor and Material Culture. Oxford, Blackwell Publishers.

Yardley, B. W. D. (1989). An Introduction to Metamorphic Petrology. Harlow, Longman Scientific \& Technical.

Zagorodny, N. y B. Balesta (2005). Estudio multidimensional de la alfarería de La Ciénaga. En M. Sempé, S. Salceda y M. Maffia (eds.), Azampay: Presente y pasado de un pueblito catamarqueño: 267-288. La Plata, Ediciones Al Margen. 
